\section{Conferences, Schools}

\section{and Programmes}

Europhysics News publishes two Meetings Issues: in March and November. Urgent up-datings can also be included in all issues in the form of advertisements charged at SFR 100-- per insertion.

The events listed here are those for which details were received at the EPS Secretariat before 5 November 1993. In general, all events interesting European physicists which take place from the beginning of 1994 are cited. For events prior to that date readers are referred to the March 1993 issue.

The meetings are in date order divided into three categories - Conferences, Schools and Programmes - although the distinction between these categories is not always evident. Europhysics Conferences, i.e. meetings organized or co-organized by EPS, its Divi-

\section{Addresses}

AAPM: Amer. Assocn. of Physicists in Medicine, $335 \mathrm{E}$ 45th St., New York, NY 10017, USA $\{+1(212)$ $6617026 /$ -

AAPT: Amer. Assocn. of Physics Teachers, 5112 Berwyn Rd., College Park, MD 20740, USA

$\{-1+1(301)$ $3454200\}$

AAS: Amer. Astro. Soc., Washington DC 20009, USA \{+1 (202) $4626903 /$ - \}

AIAA: Amer. Inst. of Aeronautics \& Aerospace, Meetings Dept., 370 L'Enfant Promenade SW, Washington, DC 20024, USA

ANS: Amer. Nuclear Soc., 555 N. Kensington Ave., La Grange Park, IL 60525, USA $\quad\{+1$ (708) 3526611 $3526464\}$

APS: Amer. Physical Soc., 335 E. 45th St., New York, NY 10017. USA $\{+1(212) 6827341 / 6872532\}$

Blois/Moriond: J. Trân Tranh Vân, LPTHE, Bât. 211, Univ. Paris-Sud, F-91405 Orsay Cedex [+33 (1) 69855483 ] 694195 51]; trantv @ frcpn11

Cargese: Institut d'Etudes Scientifiques de Cargèse (Attn.: M.-F. Hanseler), F-20130 Cargèse

$1+33(-)$

$95264121 / 95264718$ \}

CEC: Comm. of the European Communities, Euroconferences, MO75 4/9, DG-12, Rue de la Loi, 200, B-1049 Brussels $\quad\{+32$ (2) $2965171 / 2963307\}$

Courtesy Associates, Inc.: 655 15th St., NW. Suite 300 Washington DC 20005, USA

+1 (202) 6395088 $3476109]$

DBG: Deutschen Bunsen-Ges. f. Physikalische Chemie, Varrentrappstr. 40-42, D-60486 Frankfurt/Main [+49 (69) $7917201 / 7917475]$

EMRS: European Materials Res. Soc. (Attn.: P. Siffert), CEN, BP 20, F-67037 Strasbourg Cedex $2 \quad\{+33(-)$ $88286543 / 88286293$ \}

EOS: European Optical Soc., BP 147, F-91043 Orsay Cedex \{+33(1) 69853592 / 69853565$\}$

EPS: European Phys, Soc. POB 69, CH-1213 Petit. Lancy $2 \quad\{+41(22) 7931130 / 7931317\}$; epnews (6) Lancy 2 cernvm.cern.ch

ERC: European Res. Conf. (Attn.: J. Hendeković), ESF, F-67080 Strasbourg Cedex $\{+33(-) 88767135$ $88366987\}$

Erice: Ettore Majorana Centre for Scientific Culture, CCSEM, I-91016 Erice \{+39 (923) $869133 / 869226\}$ ESO: European Southern Observatory, Karl-Schwarz schild-Str. 2, D-85748 Garching bei München \{+49(89) $320060 / 32006480$ ]

Europto: Direct Communications $\mathrm{GmbH}$, Xantener Str. 22, D-10707 Berlin (+49 (30) $8805047 / 8822028$ Gordon: Gordon Res. Centre, Univ. of Rhode Island, Kingston, RI 02881-0801, USA $\{+1$ (401) 7834011$\}$ Kingston, RI 02881-0801, USA

GPS: German Phys. Soc.. Haupstr. 5, D-53604 Bad GPS: German Phys. Soc., Haupstr. 5, D- 536
Honnef $\quad\{+49(2224) 92320 / 923250\}$

Heraeus: WE-Heraeus-Stiftung, Postfach 15 53, D-63405 Hanau $\{+49(6181) 35355 / 35651\}$

IAEA: Int. Atomic Energy Agency, Cont. Service Section, POB 100, A-1400 Vienna [+43 (1) $23601310 / 234564$ \} ICTP: Int. Centre for Theo. Physics, Sci. Prog. Dept, POB 586, 1-34100 Trieste [+39 (40) 224 01/22 41 63]; postoffice (a) ictp.trieste.it

IEE: Inst. of Electrical Engineers, Cont. Services, Savoy Place, London WC2R OBL. UK (+44 (71) 3445478 $4973633\}$ sions, Groups and Sections, are printed entirely in bold type and are boxed. Events sponsored by EPS have all information printed in bold type; Europhysics Study Conferences have an $\mathbf{E}$ preceding the date and are also boxed; European Research Conferences in Physics organized in cooperation with the ESF, Strasbourg, are boxed.

Meetings sponsored by the CEC's Human Capital and Mobility programme have a $\star$ preceding the date.

For inclusion in the March 1994 issue, organizers should send details to the EPS Secretariat by 5 March 1994 at the latest. Even if full details are not yet available, the preliminary information should be given.

Entries are selected from a database compiled using information which is supplied by organizers and by meetings correspondents.
Key to Abbreviations

A: Application deadline

$\mathrm{Ab}$ : Abstracts deadline

inv.: restricted to invited applicants

NP: no proceedings will be published

P: Papers deadline (information on proceedings has not been received)

PP: proceedings will be published (papers deadline)

$x$ : no. of participants $(<x$ : limited to $x)$

c: contact (see address list "Addresses")

lim.: limited participation

: country (area) tel. / fax

email - bitnet address or other

The information is given in this order:

Date Title

Venue

Contact for information

A: .../Ab: ... / NP, PP or P: Papers deadline / Number of participants / inv. / languages other than English / conference fee Sponsor

> after the Date indicates an important amendment to the last published entry.
IEEE: Inst. of Electrical \& Electronic Engineers, Cont. Coordination, 345 E 47th St. New York, NY 10017, USA IEEE/LEOS: IEEE/Laser \& Electro-Optics Soc., 445 Hoes Lane, POB 1331, Piscataway, NJ 08855-1331, USA $\{+1$ (908) $5623893 / 5621571\}$

IMEKO: Int. Measurement Confederation, РOB 457 $\mathrm{H}-1317$ Budapest $\{+36(1) 1531562 / 1561215\}$

IOM: Inst. of Materials, Conf. Dept., 1 Carlton House Terr. London SW1Y 5BD, UK I+44 (71) $9761339 / 8231638$ IOP: Inst. of Physics, Meetings \& Conf. Dept., $47 \mathrm{Bel}$ grave Sq., London SW1X 8QX, UK \{+44(71) 2356111 . 25960 02\}; iopconf @ ulcc.ac.uk

ISF: Int. Sci. Foundation; 1054 31st St. NW, Suite 110 Washington DC 20007, USA \{+1 (202) 3422760 3422765 \}; info @ isf.org

ISPP: Int. School of Plasma Physics (Attn.: E. Sindoni) Via Celoria, 16, I-20133 Milan \{+39 (2) 2392267 23922 05\}; sindoni @ milano.infn.mi

JINR: Jont Inst for Nuclear Res. (Attn.: T. Donskova), Int Dept., POB 79, Head Post Office, CIS-101000 Dubna Russia $\{+7$ (096) $2163448 / 2166000\}$; donskova (6) ypr.jinr.dubna.su

Les Houches: Centre de Physique, Côte des Chavants, F-74310 Les Houches \{+33 (-) $50544069 / 50555325$ \} MRS: Materials Res. Soc., 9800 McKnight Rd., Pittsburg PA 15237, USA \{+1 (412) $3673003 / 3674373\}$

NATO: Scientific Affairs Division, B-1100 Brussels $\quad\{+32$ (2) $7284111 / 7284232$

OSA: Optical Soc, of America, Meetings Dept., 2010 Massachusetts Ave. NW, Washington, DC 20036-1023 USA $\quad[+1(202) 2239034 / 2236100\}$

PSJ: Physical Soc. of Japan, Kikai-Shinko Building, 3-5-8 Shiba-Koen, Minato-ku, Tokyo 105, Japan

$1+81(3)$

$34342671 / 343209$ 97)

RS: Royal Society, Meetings Sec., 6 Cariton House Terr. London SW1Y 5AG, UK [+44 (71) $8395561 / 9302170$ RSC: Royal Soc. of Chem.. Burlington House, London W1V OBN. UK \{+44 (71) $4378656 / 7341227\}$

SPIE: Soc. of Photo-Optical Instrumentation Engineers POB 10, Bellingham, WA 98227-0010, USA i+1 (206) $6763290 / 6471445$

Participation Support

(in bold: contact for general information - see "Addresses")

\begin{tabular}{|c|c|c|c|c|}
\hline Category & Funding & Availablity & Requirements & Application \\
\hline \multicolumn{5}{|l|}{ EPS meeting } \\
\hline \& sponsored meeting & Heraeus & Young scientists & Young members of GPS & GPS \\
\hline Erice school (EPS spon.) & Erice & & EPS member* & EPS \\
\hline $\begin{array}{l}\text { Euroconference } \\
\text { \& ERC }\end{array}$ & CEC & " & $\begin{array}{l}\text { Citizens of an EC country } \\
\text { or scientists resident \& } \\
\text { working there for } \geq 1 \mathrm{yr}\end{array}$ & Organizer \\
\hline $\begin{array}{l}\text { NATO ASI } \\
\text { \& ARW }\end{array}$ & NATO & Participants & $\begin{array}{l}\text { Citizens of NATO or Coop- } \\
\text { eration Partner countries }\end{array}$ & Meeting Director \\
\hline ICTP meeting & ICTP & “ & $\begin{array}{l}\text { Citizens of developing } \\
\text { countries with } \geq \text { M.Sc. } \\
+2 \text { yrs experience }\end{array}$ & ICTP \\
\hline Int. conference & ISF & " & $\begin{array}{l}\text { Scientists doing basic res. } \\
\text { in FSU or Baltic states }\end{array}$ & Organizer \\
\hline
\end{tabular}

participant or referee (can join on application)

\section{Conferences 1994}

\section{JANUARY}

Jan. 2- 9 Non-Accelerator Particle Physics: Int. Cont. Bangalore, India R. Cowsik, Indian Inst. of Astrophysics, Bangalore 560034 , India

$530583 / 533358 \mathrm{i}$ cnapp @ iiap.ernet.in

2- March $11 \quad$ Gordon Res. Confs.

2-7 Jan. Superconductivity

30 Jan. - Feb. 4 Protons \& Membrane Reactions

6-11 Feb. Macromolecular \& Polyelectrolyte Solutions

20 - 25 Feb. Polymers in Biosystems

6- 11 March Isotopes in the Physical \& Life Sciences

6 - 11 March Chemistry of Electronic Materials

4-7 \&entura, CA, USA c: Gordon A. Mondragon, Inst. de Fisica, UNIM, AP 20-364, 01000 Mexico, DF, Mexico $\quad-/+52$ (5) 6225032

9-13 Organic Nonlinear Optics: Int. Conf. (ICONO'1) Val Thorens, France F. Kajzar, DEIN/SPE/LPEM CESaclay, F-91191 Gif-sur-Yvette Cedex

$086810 / 69087679$

10-14 Millimeter \& Submillimeter Wave \& Applicns. Int. Conf. San Diego, CA, USA c: SPIE

10-15 Plasma Spectrochemistry: Winter Conf. San Diego, CA, USA R. Barnes, Chem. Dept., GRC Towers, University of Mass., Amherst, MA 01003, USA +1 (413) $5452294 / 5454490$

\section{Meetings Listings}

The Europhysics Meetings Database carries a complete listing of physics meetings worldwide of interest to European physicists. Listings sorted by date, venue and field can be ordered from the EPS Secretariat, Geneva (SFR 50.- per 50 entries)

Meetings: 682 (1994); 138 (1995)

Listed in this issue: 624 (1994/5) 
11-15 American Astro. Soc. 183rd Meeting Washington DC, USA c: AAS

17-20 Unstable Species Les Houches, France C. Demuynck, Lab. de Spectroscopie, Univ. des Sci, et Technologies de Lille, F-59655 Villeneuve d'Asq Cedex 17.22 Gross Props. of Nuclei \& Nuclear Excitations: 22nd Int. Workshop Hirschegg, Austria G. Siegrist, GSI, Postfach 1105 52, D-64220 Darmstadt +49 (6151) $359598 / 3599991$

21 LEP Performance: 4th Workshop Chamonix, France 22 - 24 Photoacoustic \& Photothermal Phenomena: Int. Conf. Pointe-à-Pitre, Guadeloup D. Fournier, ESPCI, 10, rue Vauquelin, F-75321 Paris Cedex 05 $+33(1)$ 43362395

22- 28 Superconducting Superlattice \& Multilayers Los Angeles, CA, USA 1. Bozovic, Varian Assoc. 3075 Hansen Way, MS K-114, Palo Alto, CA 94304, USA 23-26 Reactor Physics \& Reactor Computations: Int. Cont.
POB 57005, IL-61570 Tel Aviv 5612303 nerro02 @ technion.ac.il

$24-27$ Critical Currents in Superconductors: 7 th int. Workshop (IWCC-7) Alpbach, Austria A. Haber! Atominstitut, Schüttelstr. 115, A-1020 Vienna $\quad+43(1$ $217101 / 2189220$

24-28 Quantum Optics: 6th Ann. Symp. Rotorua, New Zealand D.F. Walls, Physics Dept., Auckland Univ, Private Bag 92019, Auckland, New Zealand $\quad+64$ (9) $3737999 / 3737445$ IUPAP

24-28 The Local Group - Comparative \& Global Props.: 3rd CTIO/ESO Workshop La Serena, Chile CTIO, Casilla 603, La Serena, Chile

24 - 28 Exploration of Earth \& Ecological Monitoring - Space-Based Facilities: Workshop Moscow, Russia 1. Polyansky, AASST, IKI RAN Bldg., 84/32 Profsoyuznaya St., CIS-117810 Moscow $\quad+07$ (095) 3332445 / 3301200 aberezni @ esoc1 SUS 120.-; incl. proc. 24- 28 Current Problems of Fundamental Sciences: 2nd Int. Scientific-Technical Conf. Moscow, Russia Moscow State Univ. of Tech., 2 ul. Bauman, No. 5, CIS-107005 Moscow, Russia +7 (86344) $61781 / 61877$ 24- 29 Nuclear Physics: 32nd Int. Meeting Bormio, Italy I, lori, Did. Fisica, Via Celoria, 16, I-20133 Milan +39 (2) $2392253 / 2392487$ bormio @ milano.infn.it 25-26 A Review of Results from Space: Royal Soc. Discussion Meeting on the Solar System London, UK c: RS

25 - Feb. 4 The Gama-Ray Sky with COMPTON GRO \& Sigma: NATO ASI Les Houches, France M. Signore, ENS, F-75231 Paris Cedex $05 \quad-/+33$ (1) 43367204 A: 15 Nov 93

\section{FEBRUARY}

Feb. Deep Inelastic Scattering \& Related Physics: Int. Workshop Eilat, Israel A. Levy, School of Physics, Tel-Aviv Univ., IL-69978 Tel Aviv + (3) 6408336 / 163 @ taunivm

Feb. Critique of the Sources of Dark Matter in the Universe: Int. Symp. Santa Monica, CA, USA M. Larenta, Physics Dept., UCLA, 405 Hilgard Ave., Los Angeles, LA 90024-1547, USA

larenta @ uclahep

Feb./March New Magnetic Materials: UK Seminar Sheffield, UK M. Swadling, UK Magnetics Club, Wantage Business Park, Wantage, Oxon OX12 9BJ, UK +44 (235) $770652 / 771144$

6-10 Non-Linear Optics: 3rd Franco-Israeli Symp. Ein Bokek, Israel c: SPIE

7-10 Advanced Solid-State Lasers: Topical Meeting Salt Lake City, UT, USA c: OSA

8-12 Luminescence of Porous Silicon \& Silicon Nanostructures Les Houches, France J.C. Vial, Lab. de Spectrométrie Physique, Grenoble Univ., BP 87 F-38402 St. Martin d'Hères Cedex

11- 13 Yukawa Couplings \& Their Origins: 2nd IFT Workshop Gainesville, FL, USA Y. Dixon, Physics Dept.. 247 Williamson Hall, Gainesville, FL 32611-2085, USA +1 (904) $3925707 / 3928743$ iftws @ phs.ufl.edu $12-19$ Quantum Theory: 34th Sanibel Symp. Ponte Verde Beach, FL, USA 362 Williamson Hall, Florida Univ., Gainesville, FL 32611-2085, USA +1 (904) $3921597 / 3928722$ sanibel @ qtp.ufl.edu

13-18 Smart Structures \& Materials: $1994 \mathrm{~N}$. American Conf. Orlando, FL, USA c: SPIE

14-16 Nonlinear Phenomena in Complex Systems: 3rd Ann. Seminar Polatsk, Belarus V.I. Kuvshinov, Inst. of Physics, F. Scaryna Ave. 70, CIS-220072 Minsk. Belarus +7 (0172) $394559 / 393131$ kilin @ adonis.ias.msk.su $\mathrm{Ab}$ :

incl. board, lodge; incl. proc.

14- 17 Mesoscopic Physics \& Fundamental Problems in Quantum Mechanics: Int. Conf. Rome, Italy E. Di Silvestro, Dip. di Fisica, Univ. di Roma, Ple. A. Moro, 2, 1-00185 Rome+39 (6) $49914316 / 490275$ edisilvestro @ roma1.infn.it

14-25 Meson \& Baryons in Hadronic Matter: Int. Workshop Trieste, Italy G. Siegrist, GSI, Postiach 1105 52, D-64220 Darmstadt $\quad+49(6151)$ $359598 / 3599991$
15- 18 Aerodynamics of Hypersonic Flight Vehicles \& the Physics of Hypersonic Flows: Workshop Moscow, Russia I. Polyansky, AASST, IKI RAN Bldg. 84/32 Profsoyuznaya St., CIS-117810 Moscow, Russia +07 (095) $3332445 / 3301200$ aberezni @ esoc1 16-18 Geometrische und elektronische Struktur von Oberflächen: Heraeus Seminar Bad Honnef, Germany c: GPS inv. 17-19 Integrated Photonics Res.: Topical Meeting
San Francisco, CA, USA c: OSA

18-22 Unveiling Large-Scale Structures behind the Milky Way Meudon, France C. Balkowski, Obs. de Paris-Meudon, 5, pl. Jules Janssen, F-92195 Meudon Principal Cedex + 33 (1) $45077530 / 45077893$

18 - 23 American Academy for the Advancement of Sci. Ann. Meeting San Francisco, CA, USA R. Sinclair, Phys. Div., NSF, Washington DC 20550, USA

21-25 Fibre Optic Sensing \& Environmental Monitoring: Int. Symp. Prague, Czech Republic c: Europto 21-26 Interplay of Genetics \& Physical Processes in Biological Forms Les Houches, France F. Dykstra, Atelier de Fragmentation, IPN, F-91046 Orsay Cedex

21-Dec. 2 Int. Centre for Theo. Physics: Workshops 21 Feb. - March 4 Study of Atmospheric Interactions by $\star 7.25$ March Remote Sensing

7-25 March Sci. \& Technology of Thin Films

11 April-May 13 Nuclear Reactors - Physics, Design \&

$\star 16-26$ May Commutative Algebra \& its Relation to

16 May - June 3 Air Pollution Modelling for Environ

mental Impact Assessment

13 June Search for New Elementary Particles

13 June - July 1 Res. Workshop

4-22 July Strong Correlations \& Quantum Critical

Phenomena: Miniworkshop

1.12 Aug. Non-linear Electromagnetic Interac-

$\star 6-15$ Aug. Algebraic Geometry: Adv. Workshop

$\star 10-28$ Oct. Variational \& Local Methods in the

7-18 Nov Study of Hamiltonian Systems

7-18 Nov. Three-dimensional Modelling of Seismic Wave Generation, Propag

14 Nov. - Dec. 2 High-Temperature Superconductivity Basic Activities: Experimental Workshop

Trieste, Italy c: ICTP

27 - March 4 Future Prospects for UV \& VUV Lasers Santa Barbara, CA, USA Engng Foundation, $345 \mathrm{E}$ 47th St., New York, NY 10017, USA

27-March 5 Matter under Extreme Conditions: 33. Int. Universitätswochen f. Kern- und Teilchenphysik (33. IUKT) Schladming, Austria H. Latal, Inst. f. Theo. Physik, Univ. Graz, Universitätsplatz 5, A-8010 Graz +43 (316) $3805225 / 384091$ utp @ edvz.kfunigraz.ac.at 28-March 3 Parallele Algorithmen zur Behandlung von Problemen der Feskörpermechanik: Heraeus $\mathrm{Se}$ minar Bad Honnef, Germany c: GPS inv. 28-March 6 Magnetic Multilayers \& Low-Dimensional Magnetism: Int. Symp. (Kourovka 94) Ekaterinburg, Russia A. Nosov, Inst. of Metal Physics, GSP-170 CIS-620219 Ekaterinburg, Russia $-1+7(03432) 44524$ ifmeun (8) imm.e-burg.su Ab: 1 Sep 93/PP 28 - March 6 Molecular Aspects of Confined Liquids Les Houches, France J.P. Hansen, Lab. de Physique, ENS, 46, allée d'Italie, F-69364 Lyon Cedex $07-/+33(-)$ 72728080

\section{MARCH}

March 1-4 Teilchenphysik: Fachtagung Dortmund, Germany c: GPS

1-Dec. 3 INFN Eliosatron Project: Workshops (1 - 8 March; 13 - 20 June; 12 - 20 Oct.; 26 Nov. - Dec. 3) Erice, Italy $\quad c$ : Erice

2-3 Journées des Polymères et Colloides Grenoble, France P. Lindner, Institut Laue-Langevin, BP 156, F-38042 Grenoble Cedex $9+33(-) 762071$ 11/ 76483906

7-9 Polymerphysik: Fachtagung Halle, Germany c: GPS

7.11 Extraterrestiche Forschung: Fachtagung Münster, Germany c: GPS

7-11 Kurzzeitphysik, Plasmaphysik: Fachtagung Erlangen, Germany c: GPS

7-11 Trends \& New Applications in Thin Films: 4th Int. Symp. + High Vacuum Interfaces \& Thin Films: 11 th Conf. (HVIFF-11) Dresden, Germany J. Hahn, Fach. Physik, TU Chemnitz, Oberfrohnaerstrasse 33, D-09117 Dresden

7.18 Forces in Scanning Probe Methods: NATO ASI Schluchsee, Germany H.J. Güntherodt, Physics Dept. Univ. Basel, Klingelbergstr. 82, CH-4056 Basel +41 (61) $2673768 / 3211440$

8-10 Physics with High-Energy Colliders: 22nd INS Symp. Tokyo, Japan Inst. of Nuclear Study, Tokyo Univ., 3-2-1 Midori-cho, Tanashi, Tokyo 186, Japan +81 (424) $699599 / 620775 \quad$ epnet:inshep::inssymp
8-17 Beyond Quasicrystals Les Houches, France F. Axel, Lab. de Physique de Solides, Univ. de Paris-Sud F-91405 Orsay

9-11 8th European Frequency \& Time Forum (EFTF) Weihenstephan, Germany

VDI, Graf-Recke-Str. 84

D-40239 Düsseldort +49 (211) $6214583 / 62141$

64Ab: 12 Oct 93 / PP / DM 670.-; 30.- students; incl. proc.

10-12 Industrial Applications of Positron Annihilation: Europhysics Industrial Workshop (EIW12) Oisterwijk, The Netherlands M.W.T. Purmer, Interfaculty Reactor Inst., Mekelweg 15, NL-2629 JB Delft +31 (15) $783877 / 786422$ NP/ incl. board, lodge

$\star$ 10-13 Generation \& Applicn. of Ultrashort X-ray Pulses: 1st Euroconf. Salamanca, Spain M.-J. Lecuyer, Lab. d'Optique Appliquée, ATMINES, ENSTA-Ecole Polytechnique, F-91120 Palaiseau

+33 (1) 60100318 160106085

$11 \quad$ European Phys. Res. Orgs. (EUPRO) Meeting $\begin{array}{ll}\text { Paris, France } & \text { K.H. Chang, FOM, Postbus } 3021 \\ \text { NL-3502 GA Utrecht } & +31(30) 923211 / 946099\end{array}$ 12-17 Nanophase Materials Davos, Switzerlan Engng. Foundation, 345 E 47th St., New York, NY 10017. USA

13-17 Physics of X-Ray Multilayer Structures: Topical Meeting Jackson Hole, WY, USA c: OSA 14-16 Integrated Ferroelectrics: 6th Int. Symp. Monterey, CA, USA A. Miller, Microelectronics Res. Lab., Univ. of Colorado, POB 7150, Colorado Springs, CO 80933-7150, USA $\quad-1+1$ (719) 5944257

14-17 Akustik: 20. Tagung DAGA Dresden, Germany Prof. Lenk, TU Dresden, Mommsenstrasse 13, D-01069 Dresden

14-18 58. Physikertagung der DPG mit den Fachgremien (Atomphysik, Didatik der Physik, Gravitation un Relativitätstheorie, Massenspektrometrie, Molekülphysik, Strahlenwirkung und Strahlenschutz, Theoretische und Mathematische Grundlagen der Physik, Quantentoptik/ + Energietechnik: Physikalische Grundlagen Hamburg. Germany c: GPS

14-18 Physics of the Magnetopause: Chapman Conf. San Diego, CA, USA American Geophysical Union, (202) $4626903 /$

14-18 25th Ann. Lunar \& Planetary Sci. Conf. Houston, TX, USA Lunar \& Planetary Inst., 3600 Bay Area Blvd., Houston, TX 77058-1113, USA +1 (713) $4862166 / 4862160$

17-18 Swiss Physical Soc. Spring Meeting Bern, Switzerland R. Durrer, Inst. f. Theo. Physik, Zürich Univ., Schönbe

17-19 Finnish Phys. Soc. 28th Ann. Meeting Jrvenp, Finland S. Nokkanen, POB 9, Helsinki Univ. FIN-00012 Univ. of Helsinki +358 (0) 1918437 1918366 nokkanen @ finuhcb.bitnet

20-22 Informatique et Pédagogie des Sciences Physiques: $6^{\text {mes }}$ Journées Nationales Villeneuve d'Asca,

France F.M. Blondel, Inst. Nat, de Recherche Pédagogique, Rue Gabriel Peri, F-92120 Montrouge

20-26 Atomic, Cluster \& Surface Physics: 9th Int Symp. (SASP 94) Maria Alm, Austria T. Maerk, Inst. $f$. Ionenphysik, Univ. Innsbruck, Technikerstr. 25, A-6020 Innsbruck +43 (512) 2185124 / 2185032 tilmannmaerk @ uibk.ac.at

21-23 Advances in Optical Imaging \& Photon Migration: Topical Meeting Orlando, FL, USA c: OSA

21-24 Heavy Ion Research - Space, Radioprotection \& Radiotherapy: Int. Coll. Valbonne, France Société Française de Radioprotection, BP 72, F-92265 Fontenay-aux-Roses +33 (1) 465471 39/4654 4610 $21-25$ Physik der Hadronen und Kerne: Fachtagung Munich, Germany c: GPS

21-25 Arbeitskreis Festkörperphysik mit den Fachgremien (Dünne Schichten, Dynamik und Statische Physik. Halbleiterphysik, Magnetismus, Metallphysik, Oberflächenphysik, Tiefe Temperaturen, Vakuumphysik und Vakuumtechnik, Chemische Physik, Mathematische Mo dellierung von Festkörpeigenschafen) Münster, Germany c: GPS

22-25 Three-Dimensional Optical Methods in Astrophysics Marseilles, France G. Courtes, Obs. de Marseille, 2, pl. Le Verrier, F-13248 Marseilles Cedex 4 $+33(-) 91959088 / 91621190$

23-25 Neutron Scattering Data Analysis: Workshop Didcot, UK c: IOP

23-25 Production \& Applicn. of Negative Light lons: 5th European Conf. Dublin, Ireland M. M. Mevk IRL$\begin{array}{lll} & \\ \text { Dublin } 9 & \text { Ab: } 1 \text { Dec } 93\end{array}$

24-26 Neutron Res. \& Applicns.: Int. Workshop Budapest, Hungary Roland Eötvös Phys. Soc., POB 433, H-1371 Budapest + 36 (1) $2016383 / 2018682$ $\star 27$-29 Foams: Euroconf. -, Ireland D. Weaire, Phys. Dept., Trinity Coll., IRL-Dublin 2 +353 (1) 772941 / 772694 
27 - 30 Optical Metrology \& Nanotechnology: Topical Meeting Engelberg, Switzerland J. Gobrecht, PSI $\mathrm{CH}-5232$ Villigen-PSI

Ab: 15 Nov 93 EOS

27-31 Indium Phosphide \& Related Materials: 6th Int. Conf. (IPRM-6) Santa Barbara, CA, USA c: IEEE 27-April 2 Quantenstochastik: Tagung Oberwolfach Germany Math. Forschungsinstitut, Albertstrasse 24 , D-79104 Freiburg $\quad-/+49$ (761) 272698

28 - 31 Phys. Soc. of Japan 49th Ann. Meeting Fukuoka, Japan c: PSJ

28-31 European Phys. Soc. Condensed Matter Div. 14th Gen. Conf. (GCCMD-14) Madrid, Spain J.L. de Segovia, Inst. de Cienca de Materiales, Sede A, CSIC, Serrano 144, E-28006 Madrid +34 (1) $5618806 / 4117651 \quad$ A: 20 Feb $94 /$ Ab: 1 Dec 93 / PP: 28 Mar 94 / EPTA 40200.-: 34150.- IOM's; 16000.- students; incl. proc.

28 - 31 IOP Thin Films \& Surfaces Group Conf (ISSC 10) Liverpool, UK c: IOP

28-31 Classical \& Quantum Gravity: Euroconf. Cambridge, UK Prof. Atiyah, Isaac Newton Inst. for Math. Sciences, 20, Clarkson Rd., CB3 OEH, UK +44 (223) $335999 / 330508$

+44 (223) $337899 / 337918$

29-31 Environmental Issues in Physics Education: OP Education Group Ann. Conf. Reading, UK c: IOP

\section{APRIL}

* April Journées des Actinides: $24^{\circ}$ Journée Oberburgl, Austria Dr. Kalvius, European Actinide Res, \& Dev. Soc., Physics Dept., E-16, TU-Munich, W-8046 Garching +49(89) $32092501 / 3206780 \quad$ c: P. Rogl: +43 (1) $313672500 / 3104597$

1 - 3 Ultimate Limits of Fabrication \& Measurement: NATO ARW Cambridge, UK M.E. Welland, Engng Dept., Cambridge Univ., Trumpington St., Cambridge CB2 1PZ, UK +44 (223) $332676 / 332662$

2 - $4 \quad$ Nuclear Fission \& Fission-Product Spectroscopy: Workshop Seyssins, France H. Faust, Institut Laue-Langevin, BP 156, F-38042 Grenoble Cedex 9 $+33(-) 76207111 / 76483906$

3- 8 Resonance lonization Spectroscopy \& its Applicns.: 7th Int. Symp. (RIS'94) Bernkastel-Kues, Germany H.-J. Kluge, Inst. f. Physik, Univ, Mainz Postfach 39 80, D-55099 Mainz +49 (6131) 393628 / 392991 ris94 @ vipmza.physik.uni-mainz.de Ab: 1 Feb 94 / PP / 200 IUPAP

4-7 Global Warming: 5th Int. Conf. San Francisco, CA, USA Global Warming Int. Center, Suite 335 7501 Lemont. Woodridge, IL 60517, USA +1 (708) $9101551 / 9101561$

$4-8>\quad$ Materials Res. Soc. Spring Meeting San Francisco, CA, USA c: MRS

5-7 Storage \& Recording Systems, 1994 Keele, UK c: IEE

5-8 Frontiers in Sci. \& Technology with Synchrotron Radiation: European Symp. Aix en Provence, France J. Gastaldi, CRMC2-CNRS, Campus de Luminy, Case 913, F-13288 Marseilles Cedex 09 +33 (-) 91826028 / 91826029 lelay @ mccir3.univ-mrs.fr

5-9 High Power Lasers: 1st Int. Symp. Vienna, Austria c: EUROPTO

7-8 Arbeitskreis Energie der DPG Bad Honnef, Germany c: GPS

8 The Netherlands' Phys. Soc. Spring Meeting Utrecht, The Netherlands R. Morgenstern, Vakgruep Atoomfysica, RUG, Zernikelaan 25, NL-9744 AA Groningen +31 (50) $633600 / 634003$

8-10 Photon \& Neutron Studies of Magnetic Materials: EC Network Workshop Marathon, Greece K. Trohidu, Inst. of Materials Sci., GR-153-10 Ag Paraskevi/Athens $-1+30$ (1) 6519430 popi @ leon.nrcps. ariadne-t.gr

10-14 Radiation Damage in DNA - Physics, Chemistry \& Molecular Biology: 18th L.H. Gray Conf Bath, UK P. O'Neill, Radiobiology Unit, MRC, Chilton Didcot, Oxon OX11 ORD, UK +44 (235) 834393 834918

10-14 Optical Methods in Biomedical \& Environ mental Sciences: 3rd int. Conf, on Optics within Life Sciences (OWLS III) Tokyo, Japan H. Ohzu, The Campus Corp., Babashita-cho 9, Shinjuku-ku, Toyko 162 Japan +81 (3) $32087106 / 32025482$

10-15 Elementary Particle Theory - Physics at LEP200 \& Beyond: Teupitz Workshop Hamburg. Germany U. Büchler, DESY-DIB, Notkestrasse 85 , D-22603 Hamburg +49 (40) $89983158 / 89944304$ dirbue @ dsyibm.desy.de

10-15 Experimental NMR Conf. (ENC-35) Pacific Grove, CA, USA ENC, 815 Don Gaspar Ave., Santa Fe, NM 87501, USA $\quad-/+1$ (505) 9891073

11 - 13 The Local Interstellar Medium within $300 \mathrm{Pc}$ \& Its Relation to the More Distant Gas Washington, DC, USA F. Bruhweiler, Physics Dept., Catholic Univ. of America, Washington DC 20064, USA

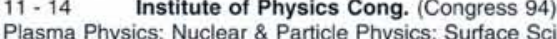
\& Technology; Atomic, Molecular \& Optical Physics Brighton, UK c: IOP

11-15 Integrated Optics: Int. Symp. Lindau, Germany c: EUROPTO Compuserve 110140,3212

11-15 Statistical Physics: 19th Int. Meeting of the Middle European Cooperation (MECO 19) Smolenice Castle, Slovakia A. Surda, Inst. of Physics, Dúbravská cesta 9, CZ-842 28 Bratislava, Slovakia +42 (7) $3782275 / 376085$ fyzisurd (9) savba.savba.sk 11-15 Nuclear Physics: 31st Holzhau Meeting Holzhau, Germany H. Prade, FZR Rossendor Inst. f. Kern- und Hadronen Physik, Postfach 510119 D-01314 Dresden +49(351) $5913270 / 5913700$

$11-15$ The Opacity of Spiral Disks: NATO ARW Cardiff, UK J.I. Davies, Dept. of Physics \& Astronomy, Univ. Coll., POB 913, Cardiff CF2 3YB, UK

11 - 151994 Int. Simulation Multiconf. (incl. High Performance Computing ' 94 : Grand Challenges in Com puter Simulation) La Jolla, CA, USA A. Tentner, ANL 9700 S. Cass Ave., Argonne, IL 60439, USA tentner @ pepper.ra.anl.gov

12-14 Computation in Electromagnetics: 2 nd Int. Conf. (CEM 94) Nottingham, UK c: IEE

12-15 Large Experiments at Low-Energy Hadron Machines: Int. Workshop Villigen, Switzerland S. Jăhrling PSI-West, $\mathrm{CH}-5232$ Villigen

993294 paris @ cvax.psi.ch

14 Complementary Neutron \& X-ray Scattering in the Study of Biological Systems: IOP Neutron Scattering Group Meeting Keele, UK c: IOP

14 Particle Physics: Conf. Bristol, UK c: IOP $14-15$ Optical Methods \& Data Processing in Heat \& Fluid Flow: Int. Seminar London, UK A. Richards, IME, 1 Birdcage Walk, London SW1H 9JJ, UK (71) 2229881

15- 17 Thin-Film Physics \& Applicns.: 2nd Int. Cont. Shanghai, PRC

$17-20 \quad$ Physics \& Chemistry of Alloy Surfaces:
Heraeus Seminar
Schloss Gaussig, German c: Heraeus

$17-22>$ Integrated Optics + Space Optics: Int Symp. (IOSO'2) Garmisch Partenkirchen, Germany c: Europto Compuserve 110140,3211

17-24 Data Analysis in Astronomy: Int. Workshop (5th Session) Erice, Italy c: Erice

18-20 Semiconductor Processing \& Characterisation with Lasers - Applicns. in Photovoltaics: 1st Int. Symp. Stuttgart, Germany M. Brieger, DLR, Inst f. Technische Physik, Pfaffenwaldring 38/40, D-70569 Stuttgart +49(711) $68620 / 6862788$

18-22 American Phys. Soc./American Assocn. of Physics Teachers: Joint Spring Meeting Crystal City VA, USA c: APS

18-22 Liquid-Particle Interactions in Suspension Flow: IUTAM Symp. Grenoble, France G. Cognet, ENSGI-INPG, 46, ave. Félix Viallet, F-38031 Grenoble Cedex $\quad-/+33(-) 76574793$

18-22 Optical Materials Technology for Energy Efficiency \& Solar Energy: Conf. Freiburg, Germany c: SPIE

20 Neutron Metrology - From Primary Measurements to Field Measurements Teddington, UK P. Kolkowski, Div. of Rad. Sci. \& Acoustics, NPL, Queen Rd., Teddington, Middlesex TW11 OLW, UK +44 (81) $9436855 / 9436161$

20-24 Superconductivity \& Particle Detection: Int. Workshop Toledo. Spain M. Fatas, Inst. de Fisica Nuclear y Altas Energias, Univ. de Zaragoza, E-50009 Saragosa $+34(76) 358321 / 568060$ fatas () gae. unizar.es A: 15 Feb 94/ Ab: 1 Mar 94/PP/inv. PTAS 30000

$21-27 \quad$ Computing in High-Energy Physics: Conf (CHEP '94) San Francisco, CA, USA M. Field, LBL 1 Cyclotron Rd., MS 50F, Berkeley, CA 94720, USA +1 (510) $4866386 / 4865548$ chep94 @ Ibl.gov 24-27 Radiation Shielding: 8th Int. Conf. Arlingtor TX, USA N.E. Hertel, Woodruff School of Mechanical Engng., GIT, Atlanta, GA 30332-0405, USA + (404) $8943717 / 8943733$

me.gatech.edu

24-29 Surface \& Interfaces in Mesoscopic Devices Kona, HI, USA New York, NY 10017, USA

25-28 Thermal Microsensors - Their Bases, Principles \& Applications: Europhysics Industrial Workshop (EIW-10) Oberhof, Germany J.Müller, Inst. für Physikalische Hochtechnologie e.V Postfach 101036 , D-07702 Jena +49(3641) 853532 / 852587 Ab: 30 Nov 93 / NP / DM 900.750.- IOM's \& AM staff; incl. board, lodge

25-29 European Geophysical Soc. 19th Gen. Ass. Grenoble, France A.K. Richter, EGS Office, Postfach 49 , D-37189 Katlenburg-Lindau +49 (5556) $1440 / 4709$ egs (a) linmpi.gwdg.de
26-27 Massively Parallel Processing using Optical Interconnections: 1st Int. Symp. + Parallel Processing: 8th Int. Symp. Cancun, Mexico E. Schenfeld, NEC Res. Inst., 4 Independence Way, Princeton, NJ 08540, USA +1 (609) $5201555 / 9512481$ IEEE 26 - 28 Neutron Spectroscopy, Nuclear Structure \& Related Topics: Int. Seminar Dubna, Russia c: JINR 27-29 Combustion Propre des Déchets et des Combustibles non Conventionnels: Journées Int. sur les Flammes Biarritz, France R. Pinatel, Elf France, Tour Elf-OSC/PCT, PI. de la Coupole, Cedex 45, F-92078 Paris La Défense $\quad \cdot /+33$ (1) 47442243

$27-29$ Artificial Neural Networks: European Symp. Brussels, Belgium Prof. Verleysen, UCL-Lab. de Microélectronique, Cath. Univ, de Louvain, PI. du Levant, 3 , B-1348 Louvain-la-Neuve +32(10) $472551 / 452272$ 28-30 Energy with All Aspects in the 21st Century: Int. Symp. Istanbul, Turkey Bulmuncu, Barbaros Blvd. 131/10, TR-80700 Besiktas/Istanbul +90 (212) $2749132 / 2882614$

\section{MAY}

May Digital Holography/Diffractive Optics: Topical Meeting Prague, Czech Republic c: EOS

May Interclass Relations: Padua Conf. on Cataclysmic Variables Abano Terme, Italy B. Bianchini, Univ. di Padova, Vicolo dell'Oss., I-35100 Padua

May High Technology Materials: European Materials Soc. Spring Meeting Strasbourg, France c: EMRS 1-5 Applied Computational Fluid Dynamics: 2nd World Conf. Basel, Switzerland B. Loeffer, POB, CH-4021 Basel $\quad-/+41$ (61) 5862186

1.5 Ultrafast Phenomena: Topical Meeting Dana Point, CA, USA c: OSA

1-6 9th Pacific Basin Nuclear Conf. (9 PBNC) Sydney, Australia Institution of Engineers, 11 National Circuit, Barton, ACT 2600, Australia -/+61(6) 2732918 1-6 Fractals: Gordon Res. Cont. San Miniato, Italy c: Gordon

2-6 Cong. Français d'Acoustique Toulouse, France Toulouse Univ., Le Mirail, 5, Allee Antonio Machado, F-31058 Toulouse Cedex

2-6 Wolf-Rayet Stars - Binaries, Colliding Winds, Evolution Elba Island, Italy K.A. van der Hucht, SRON, Sorbonnelaan 2, NL-3584 CA Utrecht - / +31 (39) $540860 \quad$ karel @ sron.ruu.nl

4-7 Medical Physics \& Biomedical Engng.: 1st Int. Conf. Nicosia, Cyprus S. Spyrou, POB 2423, Nicosia, Cyprus +357 (2) $305030 / 494953$

$\star 4-7$ Synchrotron Radiation in Biological \& Medical Sci.: Euroconference Oporto or Oeiras, Portugal A.M. Damas, Inst. de Ciencias Biomedicas, Univ. de Porto, Largo do Prof. Abel Salazar, No. 2, P-4000 Porto +351 (2) $310359 / 2001918$

8-13 Lasers \& Electro-Optics: 14 th Conf./Int. Quantum Electronics Conf. (CLEO/IQEC'94) Anaheim, CA, USA c: OSA

8-13 Phase Transitions in Non-Metallic Solids: Gordon Res. Conf. Volterra, Italy K. Schwarz, Chem. Dept., TU Wien, Getreidemarkt 9/158, A-1060 Vienna

8-21 Applicn. of Particle \& Laser Beams in Materials Technology: NATO ASI

Chalkidiki, Greece P. Misaelides, Chem. Dept., Aristotle Univ., GR-54006 Thessaloniki +30 (31) $991062 / 200802$

9-13 Nuclear Data for Science \& Technology: Int. Conf. Gatlinburg, TN, USA J.K. Dickens, ORNL. POB 2008, Oak Ridge, TN 37831, USA +1 (615) $5746133 / 5768746 \quad j k d @$ orevax.epm.ornl.gov IUPAP

9-28 Dynamics of Complex Systems in Biosciences Les Houches, France G. Weisbuch, Lab. de Physique, (1) 45873489

10-14 Systems with Fast lonic Transport: 4th Int. Symp. Warsaw, Poland W. Jakubowski, Physics Inst. , Warsaw Polytechnic, ul. Koszykowa 75, PL-00-662 Warsaw $\quad-/+48$ (2) 628171

11 8th Battery Conf. Solihull, UK Conf. Group, ERA Technology Ltd., Cleeve Rd., Leatherhead, Surrey, KT22 7SA, UK +44(372) $374151 / 377927$

12- 14 Deutsche-Bunsen Ges. f. Physikalische Chemie Hauptversammlung 1994 Berlin, Germany c: DBG $\quad$ UC

16- 19 Radiation Measurements \& Applicns.: 8th Symp. Ann Arbor, MI, USA H. Lum, 3034 Pheonix Memorial Lib., Michigan Univ., Ann Arbor, MI 48109. 2100, USA

16-19 Diffuse Interstellar Bands Boulder, CO, USA A.F.G.M. Tielens, MS 245-3, Ames Res. Center, Moffett Field, CA $94035-1000, C A$

6-20 Coronal Magnetic Energy Releases: CESRA Workshop Potsdam, Germany A. Krüger, Astrophysikalishes Inst., An de Sternwarte 16, D-14482 Potsdam 16-20 Interferometry \& Refractometry: Ann. Conf. Warsaw, Poland c: SPIE Ab: 15 Nov 93 17- 19 Soc. Française des Thermiciens: Rencontre SFT 94 Paris, France SFT, 3, rue Henri-Heine, F-75016 Paris +33 (1) $44304198 / 44304198$ 
18 Materials for Electromagnetic Compatibility 19-24 Particles, Strings \& Cosmology (PACOS 1994) Syracuse, NY, USA Phys. Dept., Syracuse Univ. Syracuse, NY 13244, USA

20-25 Correlation Effects in Molecular \& Cluster Impacts: Int. School of Solid State Physics (6th Workshop) Erice, Italy G. Benedek, Physics Dept. 2392414 A: 28 Feb 94 / PP / lim to 80 / SUS 1000 2392414 A: $28 \mathrm{Feb} 94 / \mathrm{PP} / \mathrm{lim}$. to $80 /$
incl. board, lodge; incl. proc. NATO

22- 28 Frontier Detectors for Frontier Physics: 6 th Pisa Meeting of Adv. Detectors Elba Island, Italy A. Scribano, INFN, Via Livornese 582/a, 1-56010 S. Piero a Grado +39 (50) $546111 / 589047$

23- 27 Plasma-Surface Interactions in Controlled Fusion Devices: 11th Int. Conf. Mito, Japan Nak Fusion Res. Est., JAERI, Mukoyama 801, Naka-machi, lbaraki-ken 311-01, Japan -/+81 (29) 951549

23-27 Dynamical Systems \& Chaos: Int. Con Tokyo, Japan N. Aoki, Maths. Dept., Tokyo Metropolitan Univ, 1-1 Minami-Osawa, Hachioji, Tokyo 192-03, Japan +81 (426) $771111 / 772481$

23-27 Elementary Particle Physics: 17th Kazimier Meeting Kazimierz, Poland Z. Aduk, Inst, of Theo Physics, Warsaw Univ., ul. Hoza 69, PL-00-681 Warsaw +48 (2) $6283396 / 219475 \quad$ ajduk @ fuw.edu.p A: 31 Mar 94 / Ab: 15 Apr 94 / PP: 30 Sep 94 / $100 /$ SUS 200.-; incl. board, lodge

23 - 27 Microstructure \& Phase Transition in Solids: EUROMECH Symp. Udine, Italy C. Divini, Inst. Meccanica, Univ. di Udine, Via delle Scienze, 208, 1-33100

23-28 Positron Annihilation: 10th Ann. Conf. (ICPA-10) Beijing, PRC B.-S. Cao, Physics Dept., Tsinghua Univ. Beijing 100084, PRC +86 (1) $2595861 / 2562768$ IUPAP

23-28 The Light Element Abundances: ESO/EIPC Workshop Elba, Italy c: ESO (P. Crane) 23-29 Quantum Systems - New Trends \& Methods: Workshop (QS '94) Minsk, Belarus Ya.M. Shnir, B.I. Stepanov Inst. of Physics, F. Skaryna Ave. 70, Minsk, 220602 Belarus $+7(0172) 394559 / 393131$ kilin adonis.as.msk.su PP/lim, to 150 / inv.

$24-28$ Deutschen Ges. f. angwandte Optik (DGaO) Jahrestagung 1994 Berchtesgaden, Germany L.Wan Inst. f. Angewandte Physik, TH Darmstadt, D-6428 Darmstadi $\quad+49(6151) 163017 / 164123$ lingli trudel iap.physik.th-darmstadt de

25 - 27 Heavy Ion Fusion - Exploring the Variety of Nuclear Props.: Workshop Padua, Italy A. Spalla, INFN, Via Marzola, 8, I-35131 Padua +39 (49) 831738 $844245 \quad$ spalla@ @adova.infn.it

25 - 31 Few-Body Problems in Physics: 14th Int. Conf (ICFBP-14) Willamsburg, VA, USA F. Gross, 1200 Jefferson Ave., Newport News, VA 23606, USA $-1+1$ (804) 2497002 IUPAP

25 - June 5 Int. Optometric \& Optical League Ann. Conf. Ottowa, Canada D. Leason, Int. Optometric and Optical League, 10 Knaresborough PI., London SW5 OTG, UK

26- 28 High-Temperature Superconducting Electron Devices: Int. Symp. Whistler, Canada FED, Fukide Bldg. N. 2, 4-1-21 Toranomon, Minato-ku, Tokyo 105

$\star 27$ - June 2 Nuclear \& Electron Relaxation: Cian Workshop on Magnetic Resonance San Miniato, Italy L. Claudio, Dip. di Chimica, Univ. degli Studi di Firenze, Via Gino Capponi, 7, 1-50121 Florence

$+39(55)$ $2757550 / 2757555$

29 Neutrino Physics \& Astrophysics: 16th Int. Conf. (Neutrino 94) Eilat, Israel A. Dar, Physics Dept. Technion, IL-Haifa 32000 +972 (4) $293529 / 221514$ phrl9ad @ technion

29 - June 17 Scanning Probe Microscopies \& Molecular Materials: NATO ARW Tegernsee, Germany J.P. Rabe, MPI f. Polymerforschung, Mainz Univ., Post fach 3148, D-55099 Mainz

29 - June 2 American Astro. Soc. 184th Meeting Minneapolis, MN, USA c: AAS

29 - June 6 Infrared Lasers: Topical Cont. (CIRP-6: 6th Int. Conf. on infrared Physics) Ascona, Switzerland F.K. Kneubühl, Infrared Physics Lab., ETH, HPF. Hönggerberg, $\mathrm{CH}-8093$ Zurich +41 (1) 3772340 / $3715989 \mathrm{lim}$

30-June 1 Infrared \& Raman Spectroscopy: 1st Scandinavian Symp. Bergen, Norway A. Christy, Chem. Dept., Bergen Univ., N-5007 Bergen

30 - June 1 Surface Studies of Nonlinear Lase Spectroscopies: Heraeus Seminar Kassel, Germany c: Heraeus inv.

30-June 1 Materials Testing: Int. Conf. (EUROMAT) Budapest, Hungary OMBKE, Budapest, Hungary +36 (1) $1354917 / 1561215$

30 - June 2 Atomic Spectroscopy \& Trace Analysis: 14 th Nordic Conf. Naantali, Finland A. Ivaska, Anal. Chem. Dept., Abo Akademi Univ., Biskopsgatan 8 , SF-20500 Abo Turku
30- June 3 Electromagnetic Environments \& Consequences: Int. Conf. (EUROEM 94) Bordeaux, France entre d'Etudes de Gramat, F-46500 Gramat $65105446 / 65105433$

$\star 30$-June 3 Non-linear Excitation in Biological Molecules: Euroconf. Les Houches, France M. Peyrard, Lab. de Physique, ENS, 46, allée d'ltalie, F-69364 Lyon Cedex $07+33(-) 72728374 / 72728080$

30 - June 4 Nucleus-Nucleus Collisions: 5 th Int. Conf. (ICNNC-5) Taorima, Italy M. di Toro, LNS-INFN 44. Via S. Sofia, 1-95129 Catania +39 (95) 542282 / (95) 7141815 nnconf @ Ins.infn.it IUPAP 31 - June $4 \quad$ SSC Physics \& Detectors: Int. Symp. Dubna, Russia c: JINR

31- June 6 Intersections of Particle \& Nuclear Physics: 5th Conf. St. Petersburg, FL, USA E. Driessen, TRIUMF, 4004 Westbrook Mall, Vancouver BC, V6T 2A3, Canada +1 (604) $2221047 / 2221074$ driessen @ triumfcl.bitnet

\section{JUNE}

June Spatio-Temporal Chaos \& Cellular Structures in Thermal Processes \& Convection: EUROTHERM Seminar Nantes, France H. Peerhossaini, ISITEM, La Chantrerie, CP 3023, F-44087 Nantes Cedex +33 () $40683124 / 40683199$

June Norwegian Phys. Soc. Ann. Meeting Bergen Norway G. Jarret, Physics Dept., Inst. for Energy Technology, POB 40, N-2007 Kjeller +47 (6) $806075 /$ 810920 gerd (8) ife.no

* June Many Particle Systems in Classical \& Quantum Physics: Euroconf. Luminy, France Prof. Orlandi, Dip. di Math. Pura ed Applicata, Univ. dell'Aquila, Via Vetoio, 1-67100 L'Aquila +39 (86) $2433124 / 2433180$ c: D. Testardi: +33 () 91269549 /

5-10 Solar-Terrestial Physics: 8 th Int. Symp. Sendai, Japan H. Oya, JPL, Caltech, 4800 Oak Grove Drive, Pasadena, CA 91109-8099, USA IAGA, IUPAP SGEPSS

5-10 Experimental NMR: 12th European Cont (EENC 94) Oulu, Finland L.P. Ingman, Chem. Dept. Univ. of Oulu, FIN-90570 Oulu +358 (81) 5531622 5531603 pingman @ phoenix.oulu.fi Ab: 28 Feb $94 /$ PP / FIM 1200.-; 700.-students; incl. proc.

5-9 Neural Networks: World Cong. San Diego, CA, USA Neural Network Soc., 24th St. NW. Washington, DC 20037, USA +1 (602) 466 4667/466 2888 70712.3265 @ compuserve.com

6-8 Plasma Science: Conf. Santa Fe, NM, USA A.L. Perrat, Los Alamos Nat. Lab., Group X-10, MS B-259, POB 1663, Los Alamos, NM 87545, USA +1 (505) $6671574 /$

6-9 Superconductivity: Int. Workshop Kyoto, Japan S. Tanaka, Int. Superconductivity Tech. Center, Eishin Kaihatsu Bldg., 34-3 Shimbashi 5-chrome, Minato-ku, Tokyo 105, Japan $\quad-/+81$ (3) 34314044

$6-10 \quad$ Optical Interference Coatings: Int. Symp. Grenoble, France c: EOS

6-10 Polarized Electrons \& Atoms Les Houches, France M. Leduc, Lab de Physique, ENS, 24, rue Lhomond, F-75231 Paris Cedex 05

45873489

6-11 Electron Beam Technologies: 4th Int. Cont.

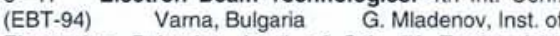
Electronics, Bulgarian Acad. of Sci., 72 Tzarigradsko Shose Blvd., BU-1784 Sofia

sabch @ gearn.bitnet

6-11 Nuclear Reaction Mechanisms: 7th Varenna Conf. Varenna, Italy E. Gadioli, Physics Dept., Via Celoria, 16, I-20133 Milan +39 (2) $2392293 / 2392487$ gadioli @ milano.infn.it

7-10 Cryogenic Engineering: 15th Int. Conf. (ICEC 15) Genoa, Italy

P. Ponta, Consorzio INFM, Via dell'Acciaio, 139, 1-16152 Genoa

6512981 infmge::consorzio

8-9 Physics \& Chemistry of Hydrogen in Metals Modelling by Muon Implantation: Disc. Meeting London, UK c: RS

10-15 Fundamental Aspects of Surface Sci.:

European Res. Conf. in Physics place undecided

c: ERC A: 15 Apr $94 / \mathrm{lim}$. CEC

$\star 11$ - 15 Neutrons in Disordered Matter: Euroconference Studsvik, Sweden U. Dahlborg, Studsvik Neutron Res. Lab., S-611 82 Nyköping +46 (15) 5221852 / 5263001

12-15 Canadian Assocn. of Physicists Cong. Regina, Canada F.M. Brûlé, Suite 903, 151 Slater St. Ottawa, Ontario K1P 5H3, Canada +1 (613) 2373392 । 2381677

12-17 Computer Coupling of Phase Diagrams \& Thermochemistry: 23rd Ann. Int. Conf. (CALPHAD-23) + Computer Applicns. to Materials \& Molecular Sci. \& Engng.: Int. Conf. (CAMSE) Madison, WI, USA Y.A. Chang, Dept. of Materials Sci. \& Engng., Univ. of Wisconsin, 1509 University Ave., Madison, WI 53706 . 1595, USA

13-15 Ocean Optics: 12 th Int. Conf. Bergen, Norway $13-17$

4th European Vacuum Conf. (EVC-4) 1 st Swedish Vacuum Meeting Uppsala, Sweden L. Westerberg, The Svedberg Lab., POB 533, S-751 21 Uppsala $+46(18) 183060 / 183833$ westerberg tsl.uu.se IUVSTA

13-17 Perspectives for the Interacting Boson Model on the Occasion of its 20th Anniversary: Int Conf. Padua, Italy J. Mooney, BNL, Bldg. 510A, Physics Dept., POB 5000, Upton, NY 11973-5000, USA $-1+1(516) 2825568$ mooney @ bnilag.bitnet 13-17 Ion Implantation Technology: 10th Int. Cont (IIT 94) Catania, Italy E. Rimini, Physics Dept., Catani Univ., Corso Italia, 57, I-95129 Catania +39 (95) $7195418 / 383023$ iit_94 @ ct.infn.it Ab: 28 Feb 94 PP / LIT 600000.-; incl. proc.

13-17 Spectral Line Shapes: 12th Int. Conf. (ICLS-12) Toronto, Canada A.D. May, Physics Dept., Toronto Univ., Toronto, M5S 1A7, Canada $-l+1$ (416) 9785848 IUPAP

13-24 Rare-Earth Doped Optical Waveguides B. Jacquier, Bât. 205, Lab. de Matériaux Lumi

$14-16$ Critical Phenomena in Polymer Physics: Conf. London, Canada A.J. Barrett, Maths \& Computer Sci. Royal Military Coll. of Canada, Kingston, Ontario K7K 5LO, Canada

14-17 Optical Pattern Recognition: Euro-American Workshop La Rochelle, France Société Française d'Optique, Inst. d'Optique, BP 147, F-91403 Paris Cedex $+33(1) 69853592 / 69853565$

\section{7 - 22 Nuclear Physics: European Res. Conf. in} Physics Helsinki, Finland c: ERC A: 15 Apr 94 lim. CEC

19-23 Quantum Chem.: 8th Int. Cong. Prague, Czech Republic

3 Dolejskova, CZ-18223 Prague 8

19-24 American Nuclear Soc. Ann. Meeting New Orleans, LO, USA c: ANS

19-24 Technology of Fusion Energy: 11th Topical Meeting New Orleans, MO, USA c: ANS

19-24 Active Phenomena in Solar System Plasma: Solar-Terrestial Physics Gordon Conf. Plymouth, NH USA M.A. Lee, Space Sci. Center, EOS, Sci. \& Engng. Res. Bldg., Univ. of New Hampshire, Durham, $\mathrm{NH}$ 03824-3525, USA

19-25 L3 Collaboration Meeting Erice, Italy c: Erice 20-23 Magnetism \& Magnetic Materials: Int. Join Conf. (MMM/INTERMAG-6)

$20-23 \quad$ 24th Int. Fluid Dynamics, Plasa Dynamics \& Laser Conf. Colorado Springs, CO, USA c: AIAA $20-23 \quad$ European Cyclotron Progress Conf. (24th ECPM) Dubna, Russia c: JINR

20-24 High Power Particle Beams: 10th Int. Cont. (Beams '94) San Diego, CA, USA A.A. Ness, Maxwell Labs, 9244 Balboa Ave. San Diego, CA 92123, USA +1(619) $5767882 / 5767659$ beams 94 ( ) scubed.com 20-24 Quark Confinement \& the Hadron Spectrum Workshop Como, Italy N. Brambilla, Dip. di Fisica Via Celoria, 16, 1-20133 Milan $\quad+39$ (2) 2392277 2392480 quarkconfin @ milano.infn.it

20-24 Sci. \& Technology of Electroceramic Thin Films: NATO ARW Villa del Mare, Italy O. Auciello, Microelectronics Center of N. Carolina, 3021 Cornwallis Rd., Res. Park Triangle, NC 27709-2889, USA

20-24 Synthesis \& Applicns. of Isotopes \& Isotopi cally Labelled Compounds: 5 th Symp. Strasbourg, France D. Blackbum, 144 Ramblewood Rd., Moorestown, NJ 08057, USA +1(609) $2351360 / 2356801$

20 - 24 Rencontre sur les Probabilités Quantiques Marseilles, France Centre Int. de Rencontres Mathématiques, Case 916, 70, route Léon-Lachamp, F-13288 Marseilles Cedex

20-24 Quantum Transport in Nanostructures: Adriatico Res. Conf. Trieste, Italy c: ICTP

21 - 23 Networks '94: Exhibition \& Conf. Birmingham, UK Blenheim Online Ltd., Blenheim House, 630 Chiswick High Rd., London W4 5BG, UK +44 (81) - / 7423182 21-24 Numerical Analysis of Semiconductor Devices \& Integrated Circuits: 10 th Int. Conf. (NASECODE X) Dublin, Ireland N. Miller, 26 Temple Lane, Dublin 2. Ireland +353(1) $6797655 / 6792469$

22-24 European Fibre Optic Communications \& Networks: 12th Ann. Conf. (EFOC \& N '94) Heidelberg, Germany c/o AKM, POB, CH-4005 Basel +41 (61) $6918888 / 6918189$

27-30 Optics \& Interferometry with Atoms: 2nd Workshop Peyresq, France Ch. Miniatura, Lab. de Physique des Lasers, Univ. Paris-Nord, Ave. J.B. Clément, F-93430 Villetaneuse +33 (1) 494034 01/49 403200 27 - July 1 Precision Elecromagnetic Measurements: Int. Conf. (CPEM '94) Boulder, CO, USA G. Bennett,
NIST, Boulder, CO 80303, USA +1 (303) 4973295 / 4976461 IUPAP 
27-July 1 4th European Particle Accelerator Conf. (EPAC'94) London, UK Ch. Petit-Jean-Genaz, CERN-AC, CH-1211 Geneva $23+41$ (22) 7673275 7678666 christin @ cernvm.cern.ch 419000 Ab: 15 Dec 93 / PP: 1 Jul $94 / 800$ / ع225.- (250. after $27 \mathrm{Apr} 94$ ); incl. proc.

27- July 1 Controlled Fusion \& Plasma Physics: 21st EPS Conf. Montpellier, France P. Platz, Bât. 507, CEA-Cadarache, F-13108 St. Paul-lezDurance +33 (-) $42256260 / 42256255$ Ab: 4 Mar 94 / PP / FF 2200.-; 2000.- IOM's, 2100.mbr. org.

27 - July 1 Real-Time Data: ESONE's 8 th Int. Cont. (RTD'94) Dubna, Russia c: JINR PP: 4 Feb 94 27-July 1 Hot Hadronic Matter - Theory \& Expt.: NATO Arizona Univ., 1118 E. 4th St., Tucson, AZ 85721, USA 27-July 1 Fusion Nuclear Technology: 3rd. Int. Symp. $27-$ July 1 Fusion Nuclear Technology: 3rd. Int. Symp
(ISFNT-3) Los Angeles, CA, USA M. Tillack, 44-139 Engineering IV, UCLA, Los Angeles, CA 90024-1597 USA +1 (310) $2961230 / 8252599$ mst @ fusion. ucla.edu 27- July 31 Mesoscopic Quantum Physics: NATO ASI
Gif-sur-Yvette, France J.L. Pichard, Serv, de Physique de l'Etat Condensé, CEN Saclay, F-91191 Gif-sur-Yvette Cedex

$\begin{array}{ll}29-\text { July } 4 & \text { World Ceramics Cong. (8th } \\ \text { Florence, italy } & \text { POB 174, 1-48018 Faenza }\end{array}$

JULY

$\star$ July Recent Trends in the Computer Simulation of Cond. Matter Systems: Euroconf. Varenna, Italy N. Palazzo, Consorzio INFN. Via Dodecaneso, 33, N. Palazzo, Consorzio INFN, Via Dodecaneso, 33,
+16146 Genoa
(10) $6520156 / 6506302$ consorzio @ infmge.ge.infn.it

Summer Physics Students: Int. Conf. Dublin, Irelan I.E. Toz, Phys. Dept., Istanbul Univ., TR-34459 Vezneciler/ Istanbul $-/+90$ (1) 5226123 icps93 @ triuvm11. bitne 1- 4 Advanced Materials in Optics, Electro-Optics \& Communication Technologies: 8th CIMTEC Conf. Florence, Italy c: EOS

1-4 Superconductivity \& Superconducting Materials Technologies: 8th CIMTEC Forum on New Materials Florence, Italy POB 174, I-48018 Faenza +39 (546) $664143 / 663362 \quad$ Ab: 15 Oct 93 / PP: 15 Apr 94

2-7 NMR Spectroscopy: 12th Int. Meeting Manchester, UK c: RSC

$\star 3-8$ Synchrotron Radiation in Materials Sci. 1st European Meeting Chester, UK G.N. Greaves, SERC Daresbury Lab., Warrington WA4 4AD, UK +44 (925) $603335 / 603174$

3-8 Liquid Crystals: 15 th Int. Conf. (ILCC-15) Budapest, Hungary L. Bata, Res. Inst. of Solid State Physics, POB 49, H-1525 Budapest - / +36 (1) 1695380 IUPAP 3.8 Synergy between Dynamics \& Reactivity at $\begin{array}{ll}3-8 & \text { Synergy between Dynamics \& Reactivity at } \\ \text { Clusters \& Surfaces: NATO ARW } & \text { Glasgow, UK }\end{array}$ Clusters \& Surfaces: NATO ARW Glasgow, UK
J.L. Farrugia, Chem. Dept., Glasgow Univ., Glasgow G12 8QQ, UK

4-7 Structure Development during Processing: Europhysics Conf. on Macromolecular Physics Eindhoven, The Netherlands P.J. Lemstra, TU Eindhoven, Postbus 513, NL-5600 MB Eindhoven $+31(40) 472840 / 546402 \quad \mathrm{PP} / 200 /$ SFR 350.-; 300.- IOM's; incl. proc.

4-8 6th Asia-Pacific Conf. (APPC-6) + Australian Inst. of Physics 11th Nat. Cong. Brisbane, Australia R. Dunlop, School of Physics, Queensland Univ. of Technology, 2 George St., GPO 2434. Brisbane, QLD 4001 Australia $\quad-/+61(7) 8641521 \quad$ r.dunlop @ qut.edu.au IUPAP

4-8 Hypernuclear \& Strange Particle Physics: Int. 4-8 Hypernuclear \& Strange Particle Physics: Int. TRIUMF, 4004 Westbrook Mall, Vancouver BC, V6T 2A3, Canada $-1+1(604) 2246910$ hyp94 (8) triumf.ca 4-8 Ceramic Oxygen lon Conductors \& their Technological Advances Lake District, UK c: IOM $\star 4$-9 Materials \& Mechanisms of Superconductivity - High-Temperature Superconductors (M2S-HTSC) Grenoble, France M. Cyrot, Lab. Louis Néel, 25, ave.
des Martyrs, F-38042 Grenoble Cedex des Martyrs, F-38042 Gren
$76881230 / 76881295$

4-9 PeH 94: Physique en Herbe - European Cong. for Young Physicists Montpellier, France E. Anglaret, +33(-) $67144677 / 67143498$ peh94@ jullienuniv. montp2.fr

4-9 Group Theoretical Methods in Physics: 20th Int Coll. (Yamada Conf.) Osaka, Japan N. Nakanishi, Res. Inst. for Mathematical Sciences, Kyoto Univ., Kitashirakawa Oiwake-cho, Sakyo-ku, Kyoto 606-01, Japan $-1+81$ (75) 7537272 noborus @ jkurims.kyoto-u.ac.jp $5-8$ Defects in insulating Materials: 7 th European Bât. 205, 43, boulevard du 11 Novembre 1918, F-69622 Bât. 205,43 , boulevard du 11 Novembre 1918 , F-69622
Villeurbane +33(-) $72448322 / 72431130$ maime
(9) pcml.univ-lyon $1 . f$

A: 1 May 94 / Ab: 1 $5-8$ Solid Int. Conf. Wroclaw, Poland W. Suski, Low Temp. \& Structure Res., POB 937, PL-50-950 Wroclaw 2 \& Structure Res., POB 937, PL-50-950 Wroclaw 2
+48 (71) $35021 / 441029$
PP: 1 May $94 / 250 /$ SUS 300.-; 200.- IOM's; 100.- students

5-9 Selected Topics in Nuclear Structure: Int. Conf. Dubna, Russia c: JINR

5-9 5th European Turbulence Conf. Sienna, Italy R. Benzi, Dip. di Fisica, Univ, di Roma "Tor Vergata", Via della Ricerca Scientifica, 1, I.00133Rome benzi (1) vaxtov.dnet.nasa.gov

6-8 Energy for the 21st Century: World Energy Res. Symp. (Flowers '94) Florence, Italy c/o Dept. of Energy Engng, Florence Univ., Via S. Marto, 3, I-550139 Florence

8-18 High-Energy Physics: QCD Workshop '94 Montpellier, France S. Narison, LPM-USTL, Place E. Bataillon, F-34100 Montpellier +33 (-) 67143568/ 67143031 narison @ cernvm.cern.ch A: 15 Mar 94 / Ab: 15 May 94 / PP: 15 May 94 / FF 1200.-; incl. proc. CNRS, NATO

9-22 Excitonic Processes in Condensed Matter: Int. Conf. (EXCON'94) Darwin, Australia J. Singh, Sci. Fac., NT Univ., POB 40146, Casurina, NT 0811, Australia $-1+61$ (89) 410460 IUPAP

10-23 Physics \& Materials Sci. of High- $\mathrm{T}_{\mathrm{c}}$ Superconductivity: NATO ASI Porto Carras, Greece N.G. Eror, Dept. of Materials Sci. \& Engng., Pittsburg Univ., Pittsburg, PA 15261, USA

11-14 Applicns of Laser Techniques to Fluid Mechanics: 7 th Int. Symp. (LADOAN-7) Lisbon, Portugal M.V. Heitor, Dept. of Mechanical Engng, Inst. Superior Técnico, Av. Rovisco Pais, P-1096 Lisbon Codex +351 (1) $8473453 / 8496156$ Ab: 17 Dec $93 / \mathrm{PP}: 15$ May 94 11-15 Climate Sensitivity to Radiative Perturbations - Physical Mechanisms \& Validation: NATO ARW Paris, France H. Le Treut, Lab. de Météorologie Dynamique, ENS, 24, rue Lhommond, F-75231 Paris I+ (1) 45873489

11-16 European Group for Atomic Spectroscopy (EGAS): 26th Conf. Barcelona, Spain R. Corbalán, Dept. de Física, Fac. de Ciencias, Univ. Autonoma de Barcelona, E-08193 Bellaterra +34 (3) $5811653 / 5812155$

11-21 COSPAR 30th Scientific Assembly Hamburg, Germany COSPAR, 51, blyd. de Montmorency, F-75006 Paris + $+33(1) 45250679 / 40509827 \quad 29531:$ cospar 12-15 Optoelectronics: 5 th Conf. (OEC'94) Chiba Japan K. Iga, Precision \& Intelligence Lab., Tokyo Inst. of Tech., 4259 Nagatsuta, Midoriku, Yokohama, 227 Japan +81 (45) $9210898 / 9225358$

12 - 15 Cooperative Phenomena in Many-electron Systems \& Their Response to External Fields: Adriatico Res. Conf. Trieste, Italy c: ICTP

17-21 Ultrasound: World Congress Sapporo, Japan M. Fukuda, Div. of Ultrasound, Sapporo Med. Coll, S-1W-16, Chuo-ku, Sapporo, Japan

17-22 Electron Microscopy: 13th Int. Cong. (ICEM-13) Paris, France Soc. Française de Microscopie Electronique, 67, rue Maurice-Gunsbourg. F-94205 Ivry-sur-Seine Cedex

18-22 Synchrotron Radiation Instrumentation: 5 th Int. Conf. (SRI-94) Stony Brook, NY, USA L. Lever BNL, Bldg. 725D, Upton, NY 11973, USA sri-94 (3) bnl.gov

18-22 Radiation Physics: 6th Int. Symp. (ISRP 6) Rabbat, Morocco M. Berrada, Lab. de Physique Nucléaire, Fac. des Sciences, Ave. Ibn Batouta, BP 1014 Rabbat, Morocco +212 (7) $778973 / 779978$ 18-22 Nuclear \& Space Radiation Effects: 31st Ann Conf. Tucson, AZ, USA T.R. Oldham, Army Res. Lab. 2800 Powder Mill Rd., Adelphi, MD 20783-1197, USA +1 (301) 3943180

18-23 Mathematical Physics: 11 th Int. Cong. (ICMP-11) Paris, France D. lagolnitzer, Physique Théor., Centre d'Etudes de Saclay, CEA, F-91191 Gif-sur-Yvette Cedex +33 (1) $69088115 / 69088120$ IUPAP, IAMP $18-30$ Physics of Highly Charged lons: NATO AS (to be confirmed) Cargese, Corsica, France c: Cargese 20-22 Polymer Spectroscopy: 11th European Symp. Madrid, Spain J.M. Pastor Barajas, Fisica de la Materia Condensada, Fac. de Ciencias, Univ. de Valladolid E-47005 Valladolid +34 (983) $4523194 / 423192$ 20-27 High-Energy Physics: 27 th Int. Conf. (ICHEP-27 27th Rochester Conf.) Glasgow, UK D.H. Saxon, Dept. of Physics \& Astronomy, Glasgow Univ., Glasgow G12 BQQ, UK $\quad-1+44$ (41) $3349029 \quad$ IUPAP

24-28 American Assocn. of Physicists in Medicine: 36 th Ann. Meeting Anaheim, CA, USA c: AAPM 24 - 29 Non-Linear Optics - Materials, Fundamentals \& Applicns.: Topical Meeting Waikola, Hawaii, USA c: OSA

24-29 Optics, Imaging \& Instrumentation: Int. Symp. (SPIE's Ann. Meeting) San Diego, CA, USA c: SPIE
24 - 30 Very High Energy Cosmic-Ray Interactions: 8th Int. Symp. (ISVHECRI) Tokyo, Japan A. Ohsawa, Inst. for Cosmic-ray Res., Tokyo Univ., Midori-machi 3-2-1, Tanashi, Tokyo 188, Japan +81 (424) 699529 623096 ohsawa (2) emsun1.icr.u-tokyo.ac.j

25-29 7th Marcel Grossmann Meeting Stanford, CA USA R. Ruffini, ICRA, Univ. "La Sapienza", Piazzale Aldo Moro, 5, 1-00185 Rome - $/+39$ (6) 4454992 IUPAP 25-30 Classical \& Quantum Billiards: Symp. rique, EPFL, CH-1015 Lausanne +41 (21) 6933411 6934444

27-Aug. 2 History of Basic Discoveries in Particle Physics: Int. Conf. Erice, Italy c: Erice 27- July 1 Superconductivity: 4 th Int. Cong. Orlando FL, USA POB 27805, Houston, TX 77227, USA +1 (713) $8952500 / 4695788 \quad 70425.501$ @ compuserve.com 31-Aug. 5 Atomic Physics: 14th int. Conf. (ICAP-14) Boulder, CO, USA D. Moreland, JILA, Univ. of Colorado, Boulder, CO 80309-0440, USA +1 (303) 4925097 4925235 IUPAP

\section{AUGUST}

Aug. 1-13 Frontiers in Particle Physics: NATO AS Cargese, Corsica, France M. Levy, IESC/Boite 230 Univ. P. \& M. Curie, 4, pl. Jussieu, F-75230 Paris Cedex 05 1. 4 Computational Physics: 3rd IMACS Int. Conf Lyngby, Denmark P.L. Christiansen, Lab. of Appl. Math. Physics, TU Denmark, DK-2800 Lyngby

2-6 American Phys. Soc. Particles \& Fields Div. 1994 Meeting Albuquerque, NM, USA S. Fields, Dept. of Physics \& Astronomy, Univ, of New Mexico, 800 Yale Blvd. NE, Albuquerque, NM 87131, USA +1 (505) $2772616 / 2771520$

3- 11

Zurich, Switzerland

Int. Cong. of Mathematicians

6- 11 Small Bodies in the Solar System \& their Interactions with the Planets Uppsala, Sweden M. Valtonen, Tuorla Obs., Turku Univ., SF-21500 Pikkio +358 (21) $2435822 / 2433767$

6-13 General Relativity: 14th int. Conf. (GR-14) Florence, Italy

7-10 Applicns. of Ferroelectrics: 9th IEEE Int. Symp. (ISAF-94) University Park, PA, USA A. Bhalla, MRL, Penn State Univ.. University Park, PA 16802, USA $1+1(814) 8642326$

8- 10 Bottom of the Main Sequence \& Beyond: ESO Workshop Garching, Germany c: ESO (C: Tinney) 8-11 Asymmetric Planetary Nebulae Haifa, Israe N. Soker, Oranim-Univ. Div., IL-36810 Tivon +972 (4) $838714 / 838738$

8-12 Adv. in Liquid Scintillation Spectrometry Int. Conf. Glasgow, UK G.T. Cook, Scottish Univ. Res. \& Reactor Centre, East Kilbride G75 0QU, UK + (3552) $23332 / 29898 \quad$ Ab: 15 Dec 93

9-12 Lasers in Surface Sci.: Adriatico Res. Cont. Trieste, Italy c: ICTP

14 - 18 High-Speed Optoelectronic Devices for Communications \& Interconnection Durham, NH, USA Engng. Foundation, 345 E 47th St., New York, NY 10017 USA

14-19 Physics of Semiconductors: 22nd int. Conf. (ICPPS-22) Vancouver, Canada R. Barrie, Physics Dept., Univ. of British Columbia, BC V6T 1Z1, Canada $-1+1(604) 8225324$ IUPAP

14-19 Magnetic Resonance in Biological Systems: Int. Conf. Veldhoven, The Netherlands A. Manders, Postbus 140, NL-5500 AC Veldhoven

14 - Sept. 3 Determination of Geophysical Parameters from Space: NATO ASI Dundee, UK A.P. Cracknell, Dept. of Appl. Physics, Dundee Univ. Dundee DD1 $4 \mathrm{HN}$, UK

15-18 Strongly Correlated Electron Systems: Int Cont. (SCES '94) Amsterdam, The Netherland A. Visser, Van der Waals-Zeeman Lab, Amsterdam Univ. Valckenierstraat 65, NL-1018 XE Amsterdam +31 (20) $5255716 / 5255788$ Ab: 1 Apr 94 / PP: 15 Jun 94; incl. proc.

15-27 Int. Astronomical Union 22nd Triennial Gen. Ass. The Hague, The Netherlands IAU, Rm. 318/31 Inst. d'Astrophysique, 98bis, blvd. Arago, F-75014 Paris +33 (1) $43258358 / 40512100$ iau @ friap51

$16-20 \quad$ Nuclear Tracks in Solids: 17 th Int. Conf. Dubna, Russia V.P. Perelygin, Int. Nuclear Track Soc. JINR, Head Post Office, POB 79, CIS-101000 Dubna, Russia +7 (08221) 621 02/ (095) 2002283 Ab: 1 Mar 94 / PP: 16 Aug 94 / SUS 320.-; 140.- students $17-20$ Structure \& Dynamics of Nonlinear Waves: IUTAM/ISIMM Symp. Hanover, Germany A. Mielke, Inst. f. Angewandte Math., Univ. Hannover, Postfach, D-30167 Hannover $\quad-/+49$ (511) 7624475

18-21 Fundamental Problems in Theo. Physics: Int Symp. Dubna, Russia c: JINR

18-26 Conceptual Models for Designing Hypertext: NATO ASI Edinburgh, UK R. McAleese, Computerbased Learning Inst., Heriot-Watt Univ., Riccarton, Edinburgh EH14 4AS, UK 
18-26 Quantum Dynamics of Simple Systems: NATO ASI Stirling, UK W.J. Firth, Dept. of Physics \& Appl. Physics, Strathclyde Univ., Glasgow G4 ONG, UK 19-24 Erice Int. Seminars (19th Sem.) Erice, Italy c: Erice

22 - 25 Physics with GeV Particle Beams: Int. Conf. Jülich, Germany Conf. Service, KFA, Inst. f. Kernphysik, D-52425 Jülich +49(2461) $613833 / 614666$ Ab: 15 Jan 94 / PP: 22 Aug 94 / DM 500.

22 - 25 Optical Computing: Int. Meeting (OC '94) Edinburgh, UK B.S. Wherrett, Physics Dept., Heriot-Watt Univ., Edinburgh EH14 4AS, UK +44 (31) 4495111 4513136 ICO

22 - 26 Physics Computing '94: 6th Joint EPS/ APS Int. Conf. on Physics Computing (PC'94) Manno, Switzerland R. Gruber, CSCS, Galleria 2, Via Cantonale, $\mathrm{CH}-6928$ Manno +41 (91) 508211 / $506711 \quad$ pc94 @ cscs.ch PP: 28 Feb $94 /$ $506711 \quad$ pc94 @
SFR 400.-; 370.- IOM's

22 - 26 Superlattices, Microstructures \& Microdevices: 7 th Int. Conf. (ICSMM-7) Banff, Alberta, Canada N. Léger, NRC, Ottawa, Ontario K1A OR6, Canada (613) $9939431 / 9579828$ IUPAP

22-26 Applied Crystallography: 16th Cont. Cieszyn, Poland D. Stroz, Inst. Fizyki i Chemii Metali, Univ. Slaski, ul. Bankowa 12, PL-40-007 Katowice 22 - 26 Theory of Fusion Plasmas: Joint VarennaLausanne Int. Workshop Varenna, Italy c: ISPP Ab: 15 Jun 94 / PP / LIT 50000

22-26 Raman Spectroscopy: Int. Conf. Hong Kong D. Lee, Chem. Dept., Hong Kong Univ. of Sci \& tech. Clearwater Bay, Hong Kong

22-27 Environmental Problems \& Scientific Education - Rio Follow-up: Int. Cont.

G. Marx, Atomic Physics Dept., Eötvös Univ., Puskin u. 5 , H-1088 Budapest +36 (1) $2667902 / 2660206$ PP SUS $400 . ;$ incl. board, lodge; incl. proc. UNESCO, ICSI, ICTP, ICPE

22 - 29 27th Congress AMPERE on Magnetic Resonance Kazan, Tatarstan N.N. Suleimanov, Zavoisky Physical-Technical Inst., Sibirsky trakt 10/7, Kazan 420029, Tartarstan +7 (08432) $760503 / 765075$ vitali @ adonis.ias.msk.su

22 - Sept. 3 Systèmes Moléculaires Organisés Cargese, Corsica, France c: Cargese lim. to 60 MRE, CNRS

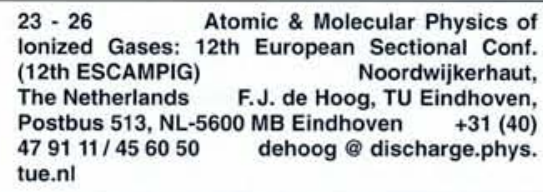

3. 26 Quantitative Surface Analysis: 8 th Int. Conf.

$\begin{array}{lll}23-26 & \text { Quantitative Surface Analysis: } 8 \text { th Int. Conf. } \\ \text { (QSA-8) } & \text { Guildford, UK } & \text { C.P. Hunt, NPL, Teddington, }\end{array}$ (QSA-8) Guildford, UK C.P. Hunt, NPL, Teddington,
Middlesex TW11 OLW, UK +44 (81) $9773222 / 9432155$ 23-27 Ferroelectricity: 5th Russian-Japanese Symp. Moscow, Russia B. Strukov, Physics Dept., Moscow State Univ., Leninskie Gory, CIS-119899 Moscow, Russia 23-27 Microstructure-Property Interactions in Composite Materials Aarlborg. Denmark R. Pyrz, Inst. of Mech. Engng., Aarlborg Univ., Pontoppidanstraede 101. DK-9220 Aarlborg $\quad-/+45$ (98) 151411

23-31 World Trends in Science \& Technology Education: 7 th IOSTE Symp. Veldhoven, The Netherlands K. Riquarts, IPN, Kiel Univ., Olshausenstr. 62, W-2300

24-26 Spectral Hole-Burning \& Related Spectroscopies - Sci. \& Applicns. Tokyo, Japan c: OSA 24-26 Effects of Organized Vortex Motion on Heat \& Mass Transfer: EUROMECH Symp. Black Sea Ukraine N.F. Yurchenko, Inst. of Hydromechanics, 8/4 Zheliabove, CIS-252057 Kiev, Ukraine

27 - Sept. 1 Electronic Structure of Solids: European Res. Conf. in Physics Gausdal, Norway c: ERC A: 15 Jun $94 / \mathrm{lim}$. CEC

28-30 History of Physical-Mathematical Sciences \& the Teaching of Physics: 2nd. Europhysics Conf. Szombathely, Hungary L. Kovács, Physics Dept., BDTF, POB 170, H-9701 Szombathely +36 (94) $13892 / 311248$

28 - Sept. 2 Lasers \& Electro-Optics: Conf. / 5th European Quantum Electronics Conf. (CLEO-Europe/ Ab: 7 Mar 94 / PP IEEE, OSA

28 - Sept. 2 Kristallographie: Fachtagung (15th European Crystallography Meeting - ECM-15) Dresden, Germany c: GPS

28 - Sept. 4

Jahn-Teller Effect: 12th Symp. Tartu, Estonia V. Hizhnyakov, Physics Inst., 142 Riaa
Str., EE-2400 Tartu
+372 (34) $28164 / 72227$ hizh@ @ark.tartu.e
29-31 Res. in High Magnetic Fields: 4th Int. Symp Nijmegen, The Netherlands J.A.A.J. Perenboom, Kath. Univ. Nijmegen, Postbus 9010, NL-6500 GL Nijmegen +31 (80) $651111 / 652120$

29 - Sept. 1 European High-Pressure Res. Group: 32nd Ann. Meeting Brno, Slovak Republic J.Vrbka, Fac. of Engng., TU Brno, Technicka 2, 61669 Brno, Czech Rep. - - I+42 (5) 41211994 vrbka @ kinf.fme.vutbr.cz NP / 150

29 - Sept. 1 Solid-State Protonic Conductors: 7th Int. Conf. (SSPC VII) Schwäbisch Gmünd, Germany MPI f. Festkörperforschung. Heisenbergerstr, 1, D-70569 Stuttgart $\quad-1+49$ (711) $6891472 \quad$ sspc \& chemix mpi-stuttgart.mpg.de PP/DM 350.-;200.-students (400.- 250.-after 1 Jul 94); incl. proc.

29 - Sept. 1 Stark korrelierte Elektronensysteme: Heraeus Seminar Schloss Ringberg, Germany c: Heraeus inv.

29 -Sept. 2 Structure of Non-Crystalline Materials: 6 th Int. Conf. (NCM-6) Prague, Czech Republic L. Cervinka, Inst. of Physics, Cukrovarnická 10, CZ-16200 Prague $6 \quad-/+42$ (2) $3123184 \quad$ IUPAP

29 - Sept. 2 Magnetic Films \& Surfaces: 14 th Int. Coll. + Magnetic Ultrathin Films, Multilayers \& Surfaces: EMRS Symp. Düsseldorf, Germany E. Kisker, Inst. Angewandte Physik, Univ. Düsseldorf, Universitätsstr. 1, W-4000 Düsseldort 1

29 - Sept. 2 Molecular Beam Epitaxy: 8th Int. Cont. (MBE-8) Osaka, Japan S. Gonda, Inst. of Sci. \& Ind. Res., Osaka Univ., 8-1 Mihogaoka, Ibaraki Osaka 567, Japan $\quad-/+81$ (6) 8776447

29 -Sept. 2 13th World Computer Cong. (IFIP Cong '94) Hamburg, Germany IFIP Sec., 16, pl. Longemalle, $\mathrm{CH}-1204$ Geneva

29 - Sept. 2 High-Speed Photography \& Photonics: Int. Cong. (ICHSPP '94) Taejon, Korea

29 - Sept 2 Mass Spectrometry: 13th Int. Conf. Budapest, Hungary Hungarian Chem. Soc., Fo u. 68 $\mathrm{H}-1027$ Budapest

29 - Sept. 2 Circumstellar Matter Edinburgh, UK G. Watt, Royal Obs., Blackford Hill, Edinburgh, EH9 $3 \mathrm{HJ}$ UK

29 - Sept. $3 \quad$ X-Ray Absorption Fine Structure: 8 th Int. Conf. (XAFS-8) Berlin, Germany F.W. Froben, Fach. Physik, Frei Univ., Arnimallee 14, D-14195 Berlin - I+49 (30) 8385567

29 - Sept. 3 Tokamak Concept Improvement: Work shop Varenna, Italy c: ISPP Ab: 15 Jun 94/PP LIT 50000 .-; incl. proc.

30 -Sept. 3 Thermoelectrics: Conf. Kansas City, MO USA B. Methiprakasam, Midwest Res. Inst., 425 Volke Blvd., Kansas City, MO 64110-2299, USA

31 - Sept. 2 French Phys. Soc. Condensed Matte Div. Conf: JMC 4 - SFP Rennes, France J. Lemaitre, $-/+33(-) 99286717 \quad$ jmc4 (a) univ.rennes1.fr 31 -Sept. $2 \quad$ Nuclear Physics: 2nd Tours Symp. Tours, France H. Utsunomiya, Physics Dept., Konan Univ., Okamoto 8-9-1. Higashinada, Kobe 658, Japan +81 (78) $4314341 / 4132672 \quad$ f51493@ @pnkudpc $\mathrm{PP} / 60$

\section{SEPTEMBER}

Autumn Particle-Solid Interactions: European
Res. Conf. in Physics (unconfirmed) San Sebastian,
$\begin{array}{ll}\text { Spain c: ERC A: } 15 \text { Jun } 94 \text { / lim. CEC }\end{array}$

$\star$ Autumn Adv. Materials - European Mater. Soc. Eurocont. Strasbourg, France c: EMRS 15-22 Polarization Phenomena in Nuclear Physics: 8th Int. Conf. + High-Energy Spin Physics: 10th Int. Conf. Bloomington, IN, USA J. Meadows, Indiana Univ. Cyclotron Fac., 2401 Milo B. Simpson Lane, Bloomington, IN 47408 , USA

spin94@ iucf.bitnet

$\star$ Sept. Euroconference at the Gran Sasso Nat. Lab. Assergi, Italy E. Bellotti, INFL, LNGS, S.S. 17 bis km.18, I-67010 Assergi +39 (8) $62437230 / 62410795$

$\star$ Sept. Magnetic Correlations, Metal-Insulator Transitions \& Superconductivity in Novel Materials: Euro conference Würzburg, Germany W. Hanke, Physikalisches Inst., Univ. Würzburg, Am Hubland,

$\star$ Sept. 27th Young Radioastronomers Conf. (YERAC) $\begin{array}{ll}\star \text { Sept. 27th Young Radioastronomers Conf. (YERAC) } \\ \text { Cambridge, UK } & \text { P. Encrenaz, Lab. de Radioastro- }\end{array}$ nomie Millimétrique, Obs. de Paris, PI. Janssen, F.92195 Meudon +33 (1) $45087911 / 45077893$ c: D.A. Green +44 (223) 354599

$\star$ Sept. Physics \& Chem. of High $\mathrm{T}_{\mathrm{c}}$ Superconductors (tentative): Euroconf. Pisa, Italy N. Palazzo, Consorzio INFN, Via Dodecaneso, 33, I-16146 Genoa +39 (10) $6520156 / 6506302$ consorzio @ infmge.ge. infn.it

$\star$ Sept. Chaos - Towards the Next Century: Euroconference Como, Italy G. Casati, Centro di Cultura Scientifica, Villa Olmo, 1, Via Cantoni, 1-22100 Como +39 (31) $572213 / 573395 \quad$ c: G. Casati: +39 (31) $3261 / 326320$

\section{$\star$ Sept.}

4th Laser Interactions Conf. (LIC 94) D. Charalambidis, FORTH, Inst. Heraktionic Structure \& Lasers, POB 1527, GR-711 10 Sept. Portuguese Phys. Soc. "Fisica '94" Biennial $\begin{array}{lll}\text { General Meeting } \quad \text { - Portugal. "Fisica '94" Biennial } & \text { C.M. Ferreira, Centro }\end{array}$ de Electrodinamica, Univ. Técnica, Inst. Superior Técnico, P-1096 Lisbon Codex +351 (1) $3524307 / 3524372$ d845 (3) alfa.ist.rccn.pt

Sept. Microstructural Evolution in Magnetic \& Magneto-Optic Thin Films: IUVSTA Workshop Crete Greece J.-E. Sundgren, IFM, Linkoping Univ., S-581 83 Linkoping $\quad-/+46(13) 137568$

Sept. Surface Sci. \& Electrochemistry: IUVSTA Workshop Ferrara, Italy K. Wandelt, Inst. f. Physikalische Chemie, Bonn Univ., Wegler Strasse 12, W-53115 Bonn $-1+49$ (228) 732551

Sept. Adv. Numerical Methods in Heat Transfer Modelling: EUROTHERM Seminar Porquerolles, France J.B. Saulnier, ENSMA, France

Sept. Anharmonic Properties of High- $\mathrm{T}_{\mathrm{c}}$ Cuprates: Workshop Bled, Slovenia D. Mihailovic, Inst. Jozef Stefan, Jamova 39, SI-61111 Ljubljana, Slovenia + +386 (61) $1259199 / 219395 \quad \mathrm{PP} / 90$

Sept. Luminescent Detectors \& Transformers of lonizing Radiation: Symp. (LUMDETR) Tallinn, Estonia G. Hûtt, Inst. of Geology, 7, Estonia Ave., EE-0105 Tallinn +372 (2) $538378 / 444189$ hutt @ pdos.gi.ee 1- 10 High-Temp. Superconductivity: 2nd. Int. Symp. Donetsk, Ukraine V.M. Svistunov, Donetsk Physical Tech. Inst., 72, R. Luxemburg St., CIS-340114 Donetsk. Ukraine +7 (062) $2557562 / 2550127$ svistuno\%ts. dipt.donetsk.ua relay.ussr.eu.net

4-8 Applied Optics \& Optoelectronics Conf. York, UK c: $10 P$

4-10 Intermittence et Structures d'Ecelles dans les Réactions à Hautes Energies Cargese, Corsica, France c: Cargese

5-7 X-Ray Topography \& High-Resolution Diffraction: 2nd European Symp. Berlin, Germany R. Köhler, MPGAG "Rotgenbeugung", Hausvogteiplatz 5-7, D-10117 Berlin 5-7 Electromagnetic Compatibility: 9 th int. Conf. Manchester, UK c: IEE

5-8 Auger Spectroscopy \& Electronic Structure: Int. Work shop (IWASES III) Liverpool, UK P. Weightman, IRC in Surface Sci., Liverpool Univ., POB 147, Liverpool L69 3BX, UK +44 (51) $7943541 / 7080662$ barbara @ ssci.liv.ac.uk $P P$

5-8 Computational Fluid Dynamics: 2nd European Conf. (ECOMAS 94) Stuttgart, Germany S. Wagner, Inst. für Aerodynamik und Gasdynamik, Univ. Stuttgart, Pfaffenwaldring 21, D-70569 Stuttgart

5-9 IMEKO 13th World Cong. Turin, Italy c: IMEKO 5-9 Active Control of Vibration: IUTAM Symp. Bath, UK C.R. Powers, School of Mech. Engng., Univ, of Bath, Claverton Down, Bath BA2 7AY, UK - / +44 (225) 826928

5-9 Multiparticle Correlations \& Nuclear Reactions: Int. Conf. (CORINNE II) Nantes, France J. Aichelin, Lab. de Physique Nucléaire, Fac. des Sciences et des Techniques, 2, rue de la Houssinière, F-44072 Nantes Cedex $03+33(-) 40374955 / 40373176$ corinne (a) frcpn 11. bitnet A: 15 Jan 94 / Ab: 15 Apr 94 / inv. 5.23 Parallel Processing \& its Applications in Physics, Chemistry \& Materials Sci.: Int. Workshop Trieste, Italy c: ICTP

6-9 Disks \& Outflows around Young Stars Heidelberg, Germany S. Beckwith, MPI f. Astronomie, D-69042 Heidelberg blythe (3) mpiahd.mpi-hd.mpg.de 6-9 Low-Temperature Physics: Int. Conf. Dubna, $\begin{array}{ll}6-9 & \text { Low-Temperat } \\ \text { Russia } & \text { c: JINR }\end{array}$

8-9 Boundary Elements \& Fluid Dynamics: Conf. Southampton, UK Wessex Inst. of Tech., Ashurst Lodge, Ashurst, Southampton SO4 2AA, UK

9-12 Physical Interpretations of Relativity Theory: Conf. London, UK M.C. Duffy, School of Engng. \& Adv. Technology, Sunderland Univ., Edinburgh Bldg.. Chester Rd., Sunderland SR1 35O, UK $5152856 / 5152703$

10-15 Electronic \& Atomic Collisions: European Res. Conf. in Physics (unconfirmed) Giens, France c: ERC A: 15 Jun $94 / \mathrm{lim}$. CEC

11- 14 Rare-Earth Magnets \& Their Applicns: 13 th Int Workshop Birmingham, UK I.R. Harris, School of Metallurgy \& Materials, Birmingham Univ., Edgbaston, Birmingham B15 2TT, UK +44 (21) $4143659 / 4145232$ 11-16 Molecular Spectroscopy: 22nd European Cong. (EUCMOS-22) Essen, Germany c: DBG

11-16 Solid-State Devices Research Conf. (ESSDERC '94) Edinburgh, UK c: IOP

11-16 Small Particles \& Clusters: 7th Int. Cont. (ISSPIC7) Kobe, Japan A. Sugano, Fac. of Sci, Himeji Inst. of tech.. Kamigori-cho, Akohgun, Hyogo 678-12, Japan 11-16 Renewable Energy: 3rd. World Cong. Reading, UK A.A.M. Sayigh, Engng. Dept, Reading Univ. POB 225, Reading RG6 2AY, UK +44 (734) 318588 । 875123 
$\star$ 11-17 Solid-State lonics: 1st Euroconf. Ionian Sea, Greece Prof. Weppner, Inst. for Solid-State Physics, Weinbergweg 20A, D-70569 Stuttgart 6875614 /

12-14 Balkan Physical Union 2nd Gen. Conf. (BPU-2) Izmir, Turkey M.N. Erduran, Cekmece Nuclear Res. Centre, PK 1, TR-34831 Havaalani +90 (1) 5484050 5482230 fkO28@ triumvm11.bitnet

12- 14 Polymer Surface \& Interfaces: Faraday Disc. 98 London, UK c: RSC

12 - 14 Large Facilities in Physics: European Phys. Soc. Conf. Lausanne, Switzerland c: EPS Phys.

12-16 Polymers, Membranes, Soft Matter: Heraeus Seminar Bad Honnef, Germany c: GPS inv.

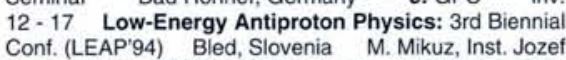
Stefan, Jamova 9, SI-61111 Ljubljana +386 (1) $159199 /$ $\begin{array}{lc}\text { Stefan, Jamova 9, SI-61111 Ljubljana } & +386(1) \\ 161029 \quad \text { marko.mikuz (B) ijs.si } & 250\end{array}$

12-19 Multiparticle Dynamics: 24th Int. Conf. Turin, Italy A. Giovannini, Dip. di Fisica Teorica, Via P. Giuria, 1, I-10125 Turin +39 (11) 6627200 6699579 vietri94 @ to.infn.it A: 15 Apr 94/ Ab: 15 Jul 94 / PP / 120 / \$ SUS 550.-; 500.- IOM's; incl. board, lodge; incl. proc.

13-14 1st Gen. Conf. of Balkan Physics Students of the BPU Students Branch Izmir, Turkey A. Dane, Physics Dept., Istanbul University, TR-34459 Vezneciler $-/+90$ (1) 5190834 fk028 @ (triuvm11.bitnet

13-17 High-Energy Physics Problems - Relativistic Nuclear Physics \& QCD: Int. Sem. Dubna, Russia c: JINR

15-18 Magnetic Anisotropy \& Coercivity in Rare-Earth Transition Metal Alloys: 8 th Int. Symp. Birmingham, UK I.R. Harris, School of Metallurgy \& Materials, Birmingham Univ., Edgbaston, Birmingham B15 2TT, UK $\quad+44$ (21) $4143659 / 4145232$

$\star 16-23$ Numerical Simulations of Quantum ManyBody Systems: Euroconf. Marciana Marina, Italy N. Palazzo, Consorzio INFN, Via Dodecaneso, 33 I-16146 Genoa

consorzio @ infmge.ge.infn.it

18-22 Aperiodic Crystals: Int. Conf. Les Diablerets, Switzerland B. Chapuis, Lausanne Univ., BSP, CH-1015 Lausanne-Dorigny

18-22 14th Int. CODATA Conf. Chambéry, France P. Glaeser, 51, Blvd. de Montmorency, F-75016 Paris +33 (1) $45250496 / 42881466$

19-21 Thermal Plasma Processes: 3rd European Cong. Aachen, Germany U. Balting, VDI-W, Graf-Recke-Str. 84, D-40239 Dûsseldort $6214254 / 6214160$

19-23 Physics of Highly Charged lons: 7th Int. Conf. ( $\mathrm{HCl}-94)$ Vienna, Austria F. Aumayr, Inst. f. Allgemeine Physik, TU Wien, Wiedner Haupstr. 8-10, A-1040 Vienn +43 (222) $588015723 / 564203$ aumayr \& eapv38 tuwien.ac.at

Surface Science: 14th European Conf. (ECOSS-14) Leipzig, Germany T. Chasse, Fab Chemie, Univ. Leipzig, Linnẻstr. 2, O-7010 Leipzig +49 (341) $6858497 / 6858225$

20 - 25 Complex Systems in Subatomic Physics: European Res. Conf. in Physics (unconfirmed) Seehiem, Germany c: ERC A: $15 \mathrm{Jun} 94 / \mathrm{lim}$. CEC

20-29 Coherence \& Turbulence: Int. Workshop Kiev, Ukraine A. Sitenko, Bogolyubov Inst. for Theo. Phy sics, 14-b, Metrolohichna St., CIS-Kiev 252143, Ukraine +7 (044) $2669108 / 2665998$ icp @ gluk.apc.org 21-26 Fundamental Physical Aspects of the Strength of Crystalline Materials: 2nd int. Cont. (JMIC-2) Strength of Materials: 10th Int. Conf. (ICSMA-10) Sendai, Japan K. Marayama, Dept. of Sci.. Tohoku Univ., Sendai 980 , Japan $-1+81$ (22) 2682949

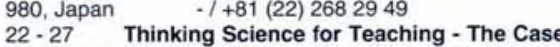
$22-27 \quad$ Thinking Science for Teaching - The Case
for Physics: Int. Conf. Rome, Italy P. Maiolo, Lab. di Didattica delle Scienze, Dip. di Fisica, Univ. "La Sapienze", P.le Aldo Moro, 2, 1-00185 Rome

Ab: 30 Nov 93 / PP: 15 Jun 94 / lim. to 100 / \$US 100. 23-28 Shape Memory Metals: Int. Symp. \& Exhibition (SMM-94) Beijing, PRC Non-Ferrous Metals Soc, of China, Beijing, PRC +86 (1) $8515368 / 8515387$

24-28 Dynamical Props. of Solids: European

Res. Conf. in Physics (unconfirmed) Castelvecchio

Pascoli, Italy c: ERC A: 15 Jun $94 / \mathrm{lim}$ CEC 24-29 Quantum Optics: European Res. Conf.
in Physics Davos, Switzerland c: ERC
$\begin{aligned} & \text { A: } 15 \text { Jun } 94 / \mathrm{lim} . \\ & \text { CEC }\end{aligned}$

26-28 Technologies de Modifications des Surfaces: $8^{\ominus}$ Conf. Int. Nice, France SF2M, 1, rue Paul Cézanne,

F-75008 Paris $\quad-/+33$ (1) 49537100
26-28 II-VI Semiconductors: European Workshop Linz, Germany H. Sitter, Inst. f. Experimentalphysik, Univ. Linz, Altenbergstr. 69, A-4040 Linz - - / +43 (732) 2468822

26- 29 Micro- \& Nano-Engineering: Cont. (MNE' 94) Davos, Switzerland P. Vettiger, IBM Res. Lab, Säumerstr. $4, \mathrm{CH}-8803$ Rüschlikon +41 (1) 7248236 / 7241789 pv@ zurich.ibm.com

26-30 Orientations in Molecule-Surface Interactions Amsterdam, The Netherlands A.W. Kleyn, FOM Inst Postbus 41883, NL-1009 DB Amsterdam +31 (20) 6081234 / $6684106 \quad$ kleyn @ amolf.amolf.n A: 15 Apr 94 / Ab: 15 Apr 94 / NP / 50 / inv. / HFL 750. incl, board, lodge CEC, ESF

$26-30 \quad$ Satellite Remote Sensing: Int. Symp. \& Exhibition Rome, Italy c: EOS

26-30 Optical Communications: 20th European Conf. (ECOC '94) Florence, Italy Ist. Internazionale delle Comunicazioni, Villa Piaggio, Via Pertinace, I-16125 Genoa +39 (10) $2722383 / 2722183$

26 - 30 Energy Transfer in Magnetohydrodynamic (MHD) Flows: 2nd Int. Conf. Aussois, France B. Collovati, LEGI-IMG, BP 53X, F-38041 Grenoble Cedex $26-31$ 18th Int. Symp. on Fusion Technology (SOFT 18) Karlsruhe, Germany

26-Oct. 1 Plasma Physics \& Controlled Nuclear Fusion Res.: 15th IAEA Conf. Madrid, Spain c: IAEA 28-29 Supersymmetry: Theory Workshop Hamburg, Germany U. Büchler, DESY-DIB, Notkestr. 85 , D-22603 Hamburg +49(40) $89983158 / 89944304$ dirbue @ dsyibm.desy.de

\section{OCTOBER}

$\star$ Oct. Spectroscopic Studies of Advanced Materials Patras, Greece G.D. Priftis, Solid State Physics Dept. Univ. of Patras, GR-26110 Patras +30 (61) 991822 /. Oct. Vibration Measurements by Laser Techniques: Topical Conf. Ancona, Italy c: EOS 2-6 European Nuclear Cong. '94 (ENC '94) Lyon, France P. Fuez, ENS, POB 5032, CH-3001 Bern +41 (31) $3206111 / 3829203$

2.7 Optical Soc. of America Ann. Meeting (OSA '94) Dallas, TX, USA c: OSA

2.7 Plasma Surface Engng.: 4th Int. Conf. Garmisch-Partenkirchen, Germany K.W. Kramer, Deutsche Ges. f. Galvano- und Oberflächentechnik, Horionplatz 6, D-40213 Düsseldorf

2 - 7 Interdisciplinary Laser Sci. Conf. (ILS-X) Dallas, TX, USA c: OSA

3-6 Charged Particle Optics: 4 th Int. Conf. (CPO4) Tsukuba, Japan JEOL Ltd., 3-1-2 Musashino, Akishima, Tokyo 196, Japan+81(425) $422244 / 463871$ 3-7 Advanced Technology \& Particle Physics: 4th Int. Conf. Como, Italy P.G. Rancoita, Physics Dept., Milan Univ., Via Celoria, 16, I-20133 Milan +39 (2) $2392331 / 2392617$ rancoita @ mi.infn.it A: 15 Jul 94 / PP: 15 May 94 / 200 / inv. / LIT 250000.-; A: 15 Jul

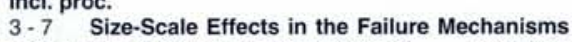
of Materials \& Structures: IUTAM Symp. Turin, Italy A. Carpinteri, Dept. of Structural Engng., Politecnico di Torino, Corso duca degli Abruzzi, 24, 1-10129 Turin $-/+39$ (11) 5644899

3. 7 Recent Developments in the Phenomenology of Particle Physics: 3rd Trieste Conf. Trieste, Italy c: ICTP

4-6 1st European Dependable Computing Conf. Berlin, Germany VDE, Stresemannallee 15, W-6000 Frankfurt/Main $70 \quad+49(69) 6308202 /$.

10-12 Electroluminescence: 7 th Int. Workshop (EL'94) Beijing, PRC S. Huab, Chanchun Inst. of Physics, 1 Yunan Rd., Changchun 130021, PRC $\quad-/+86(431)$ 55378

10-14 Nuclear Microprobe Technology \& Applicns. Int. Cont. Shanghai, PRC Z Jieqing, Shanghai Inst. for Nuclear Res., POB 800-204, Shanghai 201800, PRC $-/+86$ (21) 9528121

$11-13 \quad$ Optical Fibre Sensors: 10 th Conf. (OFS 10) Glasgow, UK A. Mitchell, Electronic \& Electrical Engng. Dept. Strathclyde Univ., Glasgow G1 1XW, UK

11 - $14 \quad$ Neutron Scattering: Int. Conf. (ICNS '94) Sendai, Japan

15-19 Materials for Microelectronics: 1st Int. Conf. Barcelona, Spain c: IOM

24-26 Coupled Cluster Methods in Physics \& Chemistry: Heraeus Seminar Bad Honnef, Germany c: GPS inv.

26-28 Plastic Optical Fibre \& Applicns.: 3rd Int. Conf. (POF'94) + Fibre Sci.: Int. Symp. (ISF' 94) Yokohama, Japan c/o AKM, POB, CH-4005 Basel +41 (61) $6918888 / 6918189$

26-29 American Phys. Soc. Nuclear Physics Div. Fall Meeting Newport News, VA, USA c: APS 30 - Nov. 4 Optical Soc. of America Ann. Meeting (OPTCON '94) Boston, MA, USA c: OSA 30 - Nov. 5 Nuclear Sci. Symp. Norfolk, VA, USA 94309, USA +1(415) $9264463 / 9263654$
31-Nov. 4 Plasma Physics: 1994 Int. Conf. (ICPP '94): Joint Conf. of the 10th Kiev Conf. on Plasma Theory +10 th Int. Cong. on Waves \& Instabilities in Plasma Foz do Iguauca, Brazil INPE, Av. dos Astronautas, 1758, CP 515, CEP 12001-970, San José dos Campos, SP, Brazil +55 (123) $418977 / 218743 \quad 94 i c p p$ (6) plasma.inpe.br IUPAP

31-Nov. 4 IEEE-LEOS Ann. Meeting Boston, MA, USA c: IEEE/LEOS

\section{NOVEMBER}

$\star$ Nov. Light on Organized Molecular Systems: Euroconf. Thessaloniki, Greece J.P. Lemaistre, Jussieu, 4, Tour 22, F-75252 Paris Cedex $05+33$ (1) $44274266 / 44273882$

7-11 American Phys. Soc. Plasma Physics Div. $\begin{array}{lll}7-11 & \text { American Phys. Soc. Plasma Physics } \\ \text { Meeting } & \text { Minneapolis, MN, USA } & \text { c: APS }\end{array}$ 10-12 European Laser Assocn. 7th Biennial Cong. Barcelona, Spain Southampton Laser Unit, Royal South Hants Hospital, Southampton SO9 4PE, UK

13-18 American Nuclear Soc. Winter Meeting Washington, DC, USA c: ANS

$14-19$ Ionizing Radiation \& Polymers (IRP 94) Guadeloupe, France A. Le Moel, CEA-CE Saclay, F-91191 Gif-sur-Yvette Cedex +33 (1) 69085485 69087963

21-24 QSO Absorption Lines: ESO Workshop Garching, Germany c: ESO (G: Meylan; gmeylan (a) eso.org) 27 - Dec. 2 American Assocn. of Physicists in Me-
dicine \& Radiological Soc. of North America Joint Meeting Chicago, IL, USA c: AAPM 28 -Dec. 2 Materials Res. Soc. Fall Meeting Boston MA, USA c: MRS

\section{DECEMBER}

5-9 Flares \& Flashes - Views from the Ground \& From Space Sonneberg, Germany H.J. Brăuer. Sterwarte Sonneberg. Sonneberg, Germany +49 (3675) $2586 / 2836$

6-9 Ultrafast Phenomena \& Its Applicns.: Adriatico Res. Cont. Trieste, Italy c: ICTP

20-22 Cond. Matter \& Materials Physics Conf. Coventry, UK c: IOP

\section{Conferences 1995}

1995 ICTSI General Assembly The Hague, The Netherlands ICSTI, 51, blvd. de Montmorency, F-75016 Paris +33 (1) $45256592 / 42151262$

$1995 \quad$ History of Physics: 2nd European Cont. Berlin, Germany F. Bevilacqua, Dip. di Fisica, Univ. di Pavia "A. Volta", Via Bassi, 6, 1-27100 Pavia +39 (382) 392495 / 392563 bevilacqua @ pavia. infn.it.bitnet 1995 Frontiers in Space-Time-Energy in Schoo Physics Education: Int. Conf. Geneva, Switzerland $\star 1995$ Ultrafast Phenomena in Semiconductors: Euroconf. Madrid, Spain J. Ryan, Clarendon Lab. Oxford Univ., Parks Rd., Oxford OX13PU, UK +44 (865) $272306 / 272400$

- Jan. 5-9 Intermediate \& High Energy Nuclear Physics: 11th Nordic Meeting Gräftávallen, Sweden P.-E. Tegnér, Physics Dept., Stockholm Univ., POB 6730 S-113 85 Stockholm +46 (8) $164686 / 347817$

8.12 American Astro. Soc. 185th Meeting Tuczon, AZ, USA c: AAS

9 - 13 Quark Matter: Int. Meeting (Quark Matter '95) Monterey, CA, USA A. Poskanzer, Bldg. 50-D, LBL, Berkeley, CA 94720, USA +1 (510) $4865618 / 4864818$ posk @ lbl.gov

14-19 American Assocn. of Physics Teachers Winter Meeting Orlando, FL, USA c: AAPT

$24-25$ Developments in High-Pressure, HighTemperature Res. \& the Study of the Earth's Interior London, UK c: RS

March Generation \& Applicn. of Ultrashort X-ray Pulses: 2nd Eurocont. Jena, Germany M.-J. Lecuyer Lab. d'Optique Appliquée, ATMINES, ENSTA-Ecole Polytechnique, F-91120 Palaiseau +33 (1) 60100318 60106085

1.2 Non-Linear Optics for Information Processing \& Communications: London, UK c: RS 16-17 Technology in the Third Millenium: Disc. Meeting London, UK c: RS

30-April 3 Res. \& Communications in Physics: 2nd Int. Conf. (RACIP2) Yokohama or Tokyo Y.-H. Ichikawa, Chubu Univ, Matsumotocho 1200, Kasugaishi 487, Japan $\quad+81(568) 511111 / 520134$ gaishi 487, Japan

April Journées des Actinides: $25^{e}$ Journée Wroclaw, Poland Dr. Kalvius, European Actinide Res. \& Dev. Soc., Physics Dept., E-16, TU-Munich, W-8046 Garching +49 (89) $32092501 / 3206780 \quad$ c: P. Rogl: +43 (1) $313672500 / 3104597$ 
3 - 7 April Atomic \& Molecular Physics: 5 th European Conf. (ECAMP V) Edinburgh, UK C.A.G. Armour, Maths Dept., Nottingham Univ. Nottingham NG7 2RD, UK +44 (602) 514922 I 514951

\section{3-7 Chemical Physics: ECAMP V Symp \\ Edinburgh, UK C.A.G. Armour, Maths Dept.,} +44 (602) $514922 / 514951$

$17-21 \quad$ Materials Res. Soc. Spring Meeting San Francisco, CA, USA c: MRS

18-21 Magnetism \& Magnetic Materials: Int. Conf (INTERMAG) San Antonio, TX, USA c: Courtesy Associates, Inc

$18-2$

American Phys. Soc. Spring Meeting Washington, DC, USA c:APS

- May World Meteorological Org. 12th World Congress Geneva, Switzerland WMO, CP 2300, ave Giuseppe Motta, $41, \mathrm{CH}-1211$ Genève $2+41$ (22) 7308111 7342326

May Isotopes: Int. Conf. Beijing, PRC W. Dexi, Chinese Nucl. Soc., POB 2125, Beijing 100822, PRC $-1+86$ (1) 9357195

$\star$ May Surface Crystallography: 5 th European Conf Kloster Banz, Germany Prof. Pendry, Imperial Coll., Prince Consort Rd., Lndon SW7 2BZ, UK $\quad+44$ (71) $2258852 / 2253165 \quad$ c: K.Müller: +49 (9131) 858402 / 858400

1.5 Particle Accelerator: 16th Biennial Cont. Dallas, TX, USA R. Briggs, SSC Lab., 2550 Beckley. meade Ave., Dallas, TX 75237, USA

17- 19 American Phys. Soc. Div. of Atomic, Molecular \& Optical Physics / Canadian Assocn. of Physicists Div. of Atomic \& Molecular Physics: Joint Meeting Toronto, Canada A.D. May, Physics Dept., Univ, of Toronto, Toronto M5S 1A7, Canada

21-26 Laser \& Electro-Optics: 15th Conf. / Quantum Electronics \& Laser Sci.: 6th Conf. (CLEO/QELS '95) Baltimore, MD, USA c: OSA

22. 26 Magnetohydrodynamic Phenomena in the Solar Atmospheres Nr. Yokyo, Japan Y. Uchida Tokyo Univ., Bunkyo-ku, Tokyo 113, Japan $\quad-/+81$ (3) 38139439

25-27 Deutsche-Bunsen Ges. f. Physikalische Chemie Hauptversammlung 1995 Bremen, Germany c: DBG

- June Many Particle Systems in Classical \& Quantum Physics: Euroconf. Luminy, France c: S. Albeverio: +49 (234) $7005599 / 7094242$

11-16 American Nuclear Soc. Ann. Meeting Atlantic City, NJ, USA c: ANS

11-16 American Astro. Soc. 186th Meeting Pittsburg, PA, USA 11-16 AAS

12. 16 Weak \& Electromagnetic Interactions in Nuclei: Int. Conf. (WEIN '95) Osaka, Japan H. Ejiri, Physics Dept., Osaka Univ., Toyonaka, Osaka 560, Japan - I+81 (6) 8457525

18-22 Crystal Growth: 11th Int. Conf. (ICCG-11) The Hague, The Netherlands Netherlands Congress Centre, Postbus 82.000, NL-2508 EA The Hague

26-30 Acoustics: 15th Int. Cong. (ICA'95) Trondheim, Norway T. Gjestland, Norwegian Inst. of Tech., N-7034 Trondheim $\quad-/+47$ (7) 591412

Summer Norwegian Phys. Soc. Ann. Meeting Tromse, Nonway G. Jarret, Physics Dept., Inst. for Energy Technology, Postboks 40, N-2007 Kjeller +47 (6) $806075 / 810920 \quad$ gerd @ barney.ife.no

- July 2-7 Controlled Fusion \& Plasma Physics:
22nd European Conf. 22nd European Conf. c: EPS

4-8 Ferroelectricity: 8th European Meeting Nijmegen, The Netherlands T. Janssen, Toemooiveld, NL-6525 ED Nijmegen +31 (80) $652995 / 553450$ $4633000 @$ hnykun11

9-14 Gravitational Lensing Melbourne, Australia R. Webster, Melboume Univ., Parkville, VI 3052, Australia $\cdot 1+61$ (3) 3474783

$17-20$ Morphology of Polymers: Europhysics Conf. on Macromolecular Physics Prague, Czech Republic F. Lednicky, Inst. of Macromol. Chem., Heyrovsky
367981

23-27 American Assocn. of Physicists in Medicine: 37th Ann. Meeting + Heatlh Physics Soc. (joint meeting) Boston, MA, USA c: AAPM

31- Aug. 4 Statistical Physics: 19th Int. Conf. (STATPHYS-19) Xiamen, PRC H. Bao-Lin, Academia Sinica, POB 603, Beijing 100080, PRC

31-Sept. 6 High-Energy Physics: Int. Europhy sics Conf. (HEP 95) Brussels, Belgium c: EPS
Aug. 6-12 American Assocn. of Physics Teachers Summer Meeting Spokane, WA, USA c: AAP 21-26 Nuclear Physics: 8th Int. Conf. (INPC-8) Beijing PRC S. Zuxun, China Inst. of Atomic Energy, POB 275 Beijing 102413, PRC +86(1) $9357787 / 9357008$ 24 - Sept. 5 Int. Comm. on Cosmic Rays: 24th Cont. Rome, Italy G. Navarra, Ist. di Fisica Generale, Univ. di Torino, Pietro Ginaria, I-10125 Torino

27 - Sept. 1 Radiation Res.: 10th Int. Cong. Würzburg. Germany GSF, D-85764 Neuherberg +49(89) $31872668 / 31873362$

29 -Sept. 2 Accelerators in Applied Res. \& Techno logy: 4th European Conf. (ECAART-4) Zurich, Switzerland H. Suter, ETH-Hönggerberg. $\mathrm{CH}-8093$ Zurich Autumn Accelerator \& Large Experimental Physics Control Systems: Int. Conf. (ICALEPCS '95) IL, USA P. Lucas, FNAL, MS 307 , POB 500, Batavia, IL 60510 USA +1 (708) $8404952 / 8408590$

Sept. High Pressure Sci. \& Tech.: 15 AIRPAT Int. Cont Warsaw, Poland S. Porowski, High Pressure Research Center, ul. Sokolowska 29, PL-01-142 Warsaw - - $1+48$ (2) 39120331

\section{Sept. Gels: Europhysics Conf. on Macro-} molecular Physics Balatonszelpak, Hungary M. Zrinyi, Inst. of Phys. Chem., Tech. Univ., H-1111 Budapest $\quad-/+$ (1) 1812173

$\star$ Sept. Magnetic Correlations, Metal-Insulator Transitions \& Superconductivity in Novel Material: Eurocont. Aachen, Germany W. Hanke, Phys kalisches Inst., Univ. Würzburg, Am Hubland, W-8700 Würzburg +49(931) $8885714 / 8885141$

$\star$ Sept. Solid-State lonics: 2nd Euroconf. - Portuga Prof. Weppner, Inst. for Solid-State Physics, Weinberg weg 20A, D-70569 Stuttgart +49 (711) $6875614 /$. 4-8 European Magnetic Materials \& Applicns. Conf. (EMMA 95) Vienna, Austria P. Grössinger, TU Wien. Wiedner Haupstr. 8-10, A-1040 Vienna +43 (1) 58801 5863191

5-8 Surface Science: 15th European Conf. (ECOSS-15) Lille, France

$25-29$ 13th Int. Vacuum Cong. \& 9th Int. Conf on Solid Surfaces Yokohama, Japan A. Yoshimori Electronic Engng. Dept., Okoyama Univ. of Sci., Okoyama 700 , Japan $-/+81$ (862) 532852

25-29 Non-Equilibrium Phenomena in Supercooled Fluids, Glasses \& Amorphous Materials: Workshop Pisa, Italy D. Leporini, Dip. di Fisica, Pisa Univ., Piazza Torricelli, 2, 1-56100 Pisa +39 (50) $45223 / 48277$

Oct. American Phys. Soc. Nuclear Physics Div. Fal Meeting Indiana, USA c: APS

9-13 Applicns. of Surface \& Interface Analysis: 6th Conf. (ECASIA '95) Montreux, Switzerland

H.J. Mathieu, Dépt. des Matériaux, EPFL, MX-C (Ecublens), $\mathrm{CH}-1015$ Lausanne +41(21) $6932962 / 6933946$ Nov. Light on Organized Molecular Systems: Euroconf. $\quad-$, Germany J.P. Lemaistre, Dépt. de Recherches Physiques, Univ. P. \& M. Curie, PI. Jussieu, 4, Tour 22, F-75252 Paris Cedex 05

$44274266 / 44273882$

5-10 Optical Soc. of America Ann. Meeting (OPTCON '95) San Jose, CA, USA c: OSA

6-9 Magnetism \& Magnetic Materials: Int. Cont. (INTERMAG-40) Philadelphia, PA, USA c: Courtesy Associates, Inc.

26 - 30 Integrated Optics \& Optical Fiber Communications: Int. Conf. (IOOC) Tokyo, Japan K. Iga, Honkomagome, Bunkyo-ku, Tokyo 113, Japan +81 (3) $58145800 / 58145823$

27 - Dec. 1 Materials Res. Soc. Fall Meeting Boston, MA, USA c: MRS

Dec. 17 - 20 Cond. Matter \& Materials Physics Conf Liverpool, UK c: IOP

\section{Schools 1994}

\section{JANUARY}

Jan. 3-21 20th IAU School for Young Astronomers Pune, India D.G. Wentzel, Astron. Dept., Univ, of Maryland, College Park, MD 20742, USA

3149067 wentzel @ astro.umd.edu

12. 19 Electroweak Interactions \& Unified Theories: Rencontres de Moriond Les Arcs, France c: Tran PP / 120 / inv. / FF 5210.-; incl. board, lodge; incl. proc. CNRS, CEA

15- 22 Geometry \& Physics: Winter School Srni, Czech Republic V. Bartik, Fac. of Electrical Engng. Czech TU, Technika 2, CZ-16627 Prague 6

22-29 Condensed Matter Physics Meeting Rencontres de Moriond Villars-sur-Ollon, Switzerland D.C. Glatti, Service de Physique de I'Etat Condensé, CE Saclay, F-91191 Gif-sur-Yvette Cedex +33 (1) $69087243 / 69978786 \quad \mathrm{PP} / 60$ / inv. / FF 5210.ncl, board, lodge; incl. proc, CNRS, CEA
22- 29 Particle Physics, Atomic Physics \& Gravitation: Rencontres de Moriond $\begin{array}{lr}\text { Switzerland c: Tran } & \text { PP / } 120 \text { / inv. / FF } 46 \\ \text { incl. board, lodge; incl. proc. } & \text { CNRS, CEA, NSF }\end{array}$ Villars-sur-Ollon,

\section{FEBRUARY}

Feb. 7-15 Neurobiology: Int. School of Biophysics (22nd Course) Erice, Italy c: Erice

13-March 25 HERCULES 1994: Neutrons \& Synchrotron Radiation for Condensed Matter Studies Grenoble, France Secrétariat HERCULES, Maison des Magistères, CNRS, 166 X, F-38042 Grenoble Cedex +33 (-) $76887986 / 76887981 \quad$ A: 1 Oct 94

14 - Dec. 9 Int. Centre for Theo. Physics: Schools 14 Feb.-March 4 Quantum Optics: Winter College 16 - 25 March Dosimetry \& Dose Reduction Techniques in Diagnostic Radiology: Training Course

11-22 April String Theory, Gauge Theory \& Quantum Gravity: Spring School \& Workshop

3 May - June 10 Quantum Phases: Spring College 13 June - July 29 High-Energy Physics \& Cosmology: Summer School

5 - 23 Sept. Medical Physics - Imaging, Instrumentation \& Dose Reduction Techniques: Winter College

26 Sept. - Oct. 14 Experimental \& Theoretical Aspects of Biomolecules: College on Biophysics

26 Sept. - Oct. 21 Microprocessor-based Real-time Control - Principles \& Applications in Physics: 3rd College Autumn Basic VLSI techniques: 3rd Course (ICTP-UNU Microprocessor lab.)

$17-8$ Oct. Physics of Archaeometry \& Preservation of Works of Art: College

24 Oct.- Nov, 11 Mathematical Ecology: 4 th Autumn Course 21 Nov, - Dec. 9 Physics \& Applications of Lasers \& Optical Fibres: Training College

Trieste, Italy c: ICTP

16-28 Advances in Quantum Phenomena: Int Course Erice, Italy E.G. Beltrametti, Physics Dept. Genoa Univ., Via Dodecaneso, 33, 1-16147 Genoa $-/+(10) 313358$ beltrametti @ genova.infn.it 20-26 Particle Physics \& Cosmology: Lake Louise Winter Inst. Lake Louise, AL, Canada A. Schaapman, Physics Dept., Alberta Univ., Edmonton, AL, T6G 2J1, Canada +1 (403) $4921052 / 4923408 \quad$ IIwl @ phys.ualberta.ac A: 17 Dec $93 /$ PP / $100 /$ SCDN 150.-: incl. board, lodge; incl. proc.

22- March 2 2nd ITEP Winter School of Physics Moscow, Russia $\quad-/+1$ (095) 1236884 itepws94 @ vxitep.itep.msk.su A: 15 Nov 93

\section{MARCH}

March - Sept. 23 Programme for Industry: Cambridge Univ.

March Image Processing

11-12 April Scanning Probe Microscopy

20-24 June Turbomachinery Aerodynamics

July ISABELLE

19-23 Sept. Scanning Electron Microscopy

Cambridge, UK A. Kent/R. Taylor, Prog. for Industry,

Cambridge Univ., 1 Trumpington St., Cambridge, CB2 1QA, UK +44 (223) $332722 / 301111$

March 1-14 Adv. Technologies in Metereology: Int. School of Meteorology (7th Course) Erice, Italy c: Erice 5-12 Electronic Props. of Novel Materials - Prog. in Fullerene Research: Int. Winterschool (IWEPNM 94) Kirchberg. Austria H. Kuzmany, Inst. f. Feskörperphysik, Univ. Wien, Strudlhofgasse 4, A-1090 Vienna +43 (1) $313673206 / 3103888$ / lim, to 100

10-11 An Intro. to Applied Non-Linear Dynamics: Bifurcations, Fractals \& Chaos in Heat Transfer \& Fluid Flow Zurich, Switzerland G. Yadigaroglu, ETH-Zentrum/CLT, CH-8092 Zurich +41 (1) 6324615 / 2622158

12 - 19 Clusters of Galaxies: Rencontres de Moriond Les Arcs, France c: Tran PP / 70 / inv. / FF 5210.incl. board, lodge; incl. proc. CNRS, CEA

14-18 Multiphase Flow \& Heat Transfer: Bases, Modelling \& Applicns. Zurich, Switzerland G. Yadigaroglu, ETH-Zentrum/CLT, CH-8092 Zurich +41 (1) $6324615 / 2622158$

17 - 29 High-Energy lon Beams - Novel Beam Techniques \& Their Applicns. JSP-KEK Int. Spring School Tsukuba, Japan Y. Mori, KEK, Oho-1-1, Tsukuba-shi, Ibaraki-ken 305, Japan +81 (2) 298645209 / $298643182 \quad$ moriy @ jpnkekvx

19-26 QCD \& High-Energy Hadronic Interactions: Rencontres de Moriond Les Arcs, France c: Tran PP / 120 / inv. / FF 5210.-; incl. board, lodge; incl. proc. CNRS, CEA

19-30 Exotic Atoms \& Weak Interactions: Int. School of the Physics of Exotic Atoms (6th Course)

Erice, Italy c: Erice

21-26 Plasma Astrophysics: 24th Saas Fee Adv, Course Les Diablerets, Switzerland A.O. Benz, Astronomy Inst., ETH-Zentrum, Rămistr. 101, $\mathrm{CH}-8006$ Zurich +41 (1) $2562211 /$.

\section{APRIL}

April 18-11 Materials Res. with Nuclear Techniques: Spring School Bad Honnef, Germany c: GPS 
19-30 Physics \& Techniques of Extremely LowEnergy Beams: Int. School of the Physics of Exotic Atoms (7th Course) Erice, Italy c: Erice

28- May 5 Cyclotrons, Linacs \& Their Applicns. CERN Accelerator School Hulpe, Belgium S. von Wartburg, CERN, CH-1211 Geneva $23-/+41$ (22) 7824836 casbel @ cernvm.cern.ch A: 1 Feb 94 / inv. / BF 52000 -; incl. proc.

\section{MAY}

$\star$ May 7-18 Currents High-Energy Astrophysics: Int School of Cosmic-Ray Astrophysics (9th Course) Erice, Italy c: Erice NATO

$\star 9-20$ Lasers \& Applicns.: 2nd EPS Southern School Elundra, Crete C. Fotakis, FORTH-ISL, POB 1527, GR-711 10 Heraklion +30 (81) 210035 / $239735<40 /$ inv. CEC 15- 26 Core Level Spectroscopies for Magnetic
Phenomena - Theory \& Experiments: Int. School of Phenomena - Theory \& Experiments: Int. School of
Solid State Physics (5th Course) Erice, Italy F. Parmigiani, CISE SpA, POB 12081, 1-20134 Milan +39 (2) $21672354 / 21672620 \quad 210$ julio @ imicise.cise.it A: $28 \mathrm{Feb} 94 / \mathrm{PP} / \mathrm{lim}$. to $80 /$ SUS 1000.-; incl. board, lodge; incl. proc. NATO $18-26$ Synchrotron Radiation in Natural Sci.: Int School \& Symp. (ISSRNS-2) Jaszowiec, Poland K. Lawniczak-Jablonska, Inst. of Physics, al. Lotnikow 32/46, PL-02-668 Warsaw - / +48 (22) 430926 jablo (8) ifpan.edu.pl A: 20 Mar 94/ Ab: 15 Jan 94/PP: 18 May 94 / 120 / SUS 90.- (110.- after 20 Mar 94)

$\star 23-27$ Lasers for Medical \& Industrial Applicns. Workshop Elounda, Crete C. Fotakis, FORTH-ISL, POB 1527, GR-711 10 Heraklion $+30(81) 210035 / 239735$ 27 May - June 5 Crystallography of Molecular Biology: Int. School of Crystallography (21st Course) Erice, Italy L. Riva, Piazza Porta San Donato, 1, 1-40126 Bologna +39 (51) $243556 / 243336$ t54bom12 @ icineca.bitnet A: 30 Nov 93 / PP / \$ US 1200.-; incl. board, lodge; incl. proc.

\section{JUNE}

June 6-17 The Cosmic Dust Connection: Int. School of Space Chem. (3rd Course)

Erice, Italy J.M. Greenberg. Huyens Lab, POB 9504, NL-2300 RA Leiden $\quad+31$ (71) $275894 / 275819 \quad$ A: 1 Mar 94 PP / \$US 900.-; incl. board, lodge; incl. proc. NATO $\star 19-25>$ Heart of the Matter-From Nuclear Interactions to Quark-Gluon Dynamics: Rencontres de Blois Blois, France c: Tran PP/ $160 /$ inv. / FF 5210 incl. board, lodge; incl. proc. CEC, CNRS, CEA, MESP 20-24 Rencontre sur les Probabilités Quantiques Marseilles, France Centre Int. de Rencontres MathéMarseilles, France Centre Int. de Rencontres Mathématiques, Case 916,7
Marseilles Cedex 9

20 - July 2 Lasers \& Their Applicns. Cargese, Corsica, France c: Cargese

28 - July 8 Quantum Groups \& Integrable Systems: Int. School of Physics "Enrico Fermi" (1st Course)

Varenna, Italy L. Castellani, Dept. of Theo. Physics, Via P. Givria, 1, 1-10125 Turin +39 (11) 6527206 6699579 castellani@ vaxto.infn.it A: 1 May 94 PP / 50 / LIT 1700000.-; incl. board, lodge SIF 28 - July 29 Mesoscopic Quantum Physics: Les Houches Summer School in Theo. Physics (Session 61) Les Houches, France c: Les Houches A:1 Mar 94 $\mathrm{PP} / 50$ / inv. French / FF 4600.-; incl. board, lodge; incl. proc. NATO, CNRS, CEA

\section{JULY}

$\star$ July 3-11 From Superstring to Present-day Physics: Int. School of Subnuclear Physics (32nd Course) Erice, Italy c: Erice

4-8 Nonlinear Aspects of Physicochemical Phenomena: 9th Europhysics Summer School on Chemical Physics + Computational Chemistry: 5th Int. School Girona, Spain F. Sagués, Dept. de Química Física, Barcelona Univ., Diagonal 647 E-08028 Barcelona +34 (3) $4021222 / 4111492$ A: 31 Dec 93 / NP / 50 / SUS 200.-; 150.- IOM's, students

4-16 Mobile Particulate Systems: NATO ASI Cargese, Corsica, France c: Cargese

10-21 31st Culham Plasma Physics School Culham, UK J. Stimson, BIdg. E5, Culham Lab. Abingdon, Oxon OX14 3DB, UK $-/+44$ (235) 463288 11- 15 Electron Crystallography Theory \& Practice: Summer School Bristol \& Bath, UK J.W. Steeds, Summer School Bristol \& Bath, UK J.W. Steeds,
Physics Dept., H.H. Wills Lab, Tyndall Ave., Bristol BS8 Physics Dept., H.H. Wills Lab, Tynd
1TL, UK $\quad-/+44$ (292) 251295

$12-22$ Biomedical Applicns. of Synchrotron Radiation: Int. School of Physics "Enrico Fermi" (2nd Course) Varenna, Italy 1-00044 Frascati burattini @ irmlnf incl. board, lodge
14- 25 Int. School of Materials Sci. \& Technology: (29th Course) + Pseudomorphic HEMT Technology \& Applicns. (30th Course) Erice, Italy c: Erice

$\star 20-30$ Cellular Automata, Aggregation \& Growth Adv. Study Inst. Cambridge, UK Prof. Atiyah, Isaac Newton Inst. for Math. Sciences, 20, Clarkson Rd., CB3 OEH, UK +44 (223) $335999 / 330508$ c: A.J. McKane: +44 (61) $2754192 / 2754218$

26- Aug. 5 Observation \& Prediction of Phase Transitions in Complex Fluids: Int. School of Physics "Enrico Fermi" (3rd Course) Varenna, Italy M. Baus, Physique Statistique, Plasma, Univ. Libre de Bruxelles, CP 231, B-1050 Brussels +32 (2) $6505809 / 6505824$ A: 1 May 94 / PP / 50 / LIT 1700000.-; incl. board, lodge SIF 29-30 Theo. Physics: Summer School Paris, France

\section{AUGUST}

Aug. 2 - Sept. 9 Fluctuating Geometries in Statistical Mechanics \& Field Theory: Les Houches Summer School in Theo. Physics (session 62) Les Houches, France c: Les Houches A: 1 Mar $94 / \mathrm{PP} / 50 /$ inv. French / FF $5600 .-$; incl. board, lodge; incl. proc. NATO, CNRS, CEA

14- 20 Neutron Scattering: 2nd Summer School Zuoz, Switzerland R. Bercher, PSI, CH-5232 Villigen PS +41 (56) $993402 / 993294$ A: 30 Jun 94 / lim. to 100 $=$ SFR 450 .

28-Sept. 3 Seismic Modelling of Earth Structure: Int. School of Solid -Earth Geophysics (8th Course) Erice, Italy c: Erice

\section{SEPTEMBER}

Sept. 1 - 7 Symmetry \& Structural Props. of Condensed Matter: Int. Summer School on Theo. Physics (SSPCM'94) Poznan, Poland W. Florek, Scientific Soc., ul. Jana Matejki 48/49, PL-60-769 Poznan + 48(61) $668651 /$ sspcm @ plpuam11. bitnet A: 1 Jun 94 / Ab: 15 Apr 94 / PP / SUS 325.-; 275.- IOM's; 150.- students; 275.- IOM's, 150.- students; incl. board, lodge; incl. proc.

4-15 Current Topics in Astrophysics: Int. School of Astrophysics "D. Chalonge" (3rd Course) Erice, Italy c: Erice

$5-14$ Trends in Nuclear Physics: 29th Zakopane School of Physics Zakopane, Poland W. Meczynsk Inst. of Nuclear Physics, ul. Radzikowskiego 152, PL-31 342 Cracow +48 (12) $370222 / 371881$ meczynski @ vsb01.ifj.edu.pl

$\star 11$ Plasma Astrophysics: European Astrophysica Doctoral Network Summer Schools Cortona, Italy C. Ray, School of Cosmic Physics, 5 Merrion Sq., IRLDublin $2+353(1) 774321 / 682003 \quad$ c: C. Chiuuder: +39 (55) $27521 / 220039$

12-16 Exotic Beams: Euroschool Leuven, Belgium M. Huyse, Inst. voor Kerm- en Stralingsfysika, Kath. Univ. Leuven, Celestijnenlaan 200D, B-3001 Leuven +32 (16) $201015 / 291959$

15-23 Electromagnetic Probes of the Structure of Hadrons \& Nuclei: Int. School of Nuclear Physics (16th Course) Erice, Italy A. Faessler, Inst. f. Theo. Physik, Univ. Tübingen, Auf der Morgenstelle 14, D-72076 Tübingen $+49(7071) 296370 / 296400$ faessler (G) mailserv. zdf uni-tuebingen de

18 - 30 Supramolecular Structure \& Function: 5 th int Summer School in Biophysics Rovinj, Istria, Croatia G. Pifat-Mrzljak, Ruder Boskovic Inst., Bijenicka cesta 41001 Zagreb, Croatia $\quad+385$ (41) $461111 / 434467$
A: 31 Dec 93 / Ab: 1 Jun 94 / PP / 120/ DM 850,-; incl. board, lodge UNESCO, IUPAP

19-28 Radiopropagation - Physics \& Applens. 10th Bodrum School of Physics Bodrum, Turkey S. Radicella, ICTP, POB 586, I-34100 Trieste +39 (40) $22401 / 224163$ rsandro @ ictp.trieste.it SUS $350 .-$; incl. board, lodge; incl. proc.

19-30 An Introduction to Accelerator Physics: CERN Accelerator School Vienna, Austria S. von Wartburg, $\mathrm{CH}-1211$ Geneva $23-1+41$ (22) 7824836 casaus (3) cernvm.cern.ch A: 1 Feb 94 / inv./ BF 52000 ; incl, proc. 25-30 New Developments in Semiconductor Physics: Int. Summer School Balatonfüred, Hungary Roland Eötvōs Phys. Soc., POB 433, H-1371 Budapest +36 (1) $2016383 / 2018682$

\section{OCTOBER}

$\star$ Oct. 1- 15 Nonlinear Superconducting Devices \& High-T Materials Capri, Italy Prof. Christiansen, Appl. Mathematical Phys. Dept., TU Denmark, Bldg. 303 DK-2800 Lyngby +45 (-) $42883715 / 4593135$ c: R.D. Parmentier: +39 (89) $822213 / 953804$

10-14 Plasma Physics: Summer Univ. Garching, Germany W. Dyckhoff, IPP, Boltzmannstr. 2. D-85740 Garching $\quad$ - / +49 (89) 32991001

$\star 20-31 \quad$ Electro-optical Techniques: Int. School of Quantum Electronics (19th Course) Erice, Italy c: Erice 20 - Nov. 14 Adv. Technologies in Hydrometeorology: Int. School of Meteorology (8th Course) Erice, Italy c: Erice

\section{DECEMBER}

$\star$ Dec. $6-17 \quad$ Astrophysics: Canary Islands Winter School Tenerife, Canary Islands F. Sanchez, Inst. de Astrofisica, Via Lactea S/N, ES-38200 La Laguna +34 (922) $605272 / 605210$

\section{Programmes 1994}

Jan. - Jun Isaac Newton Inst. for Math. Sci. Geometry \& Gravity

Cellular Automata, Aggregation \& Growth

Cambridge, UK P. Goddard, DAMTP, Cambridge Univ. Silver St., Cambridge CB3 9EW, UK +44 (223) 337883 \% pg1 @ ukacrl

Jan-July Inst. for Theo. Physics

Jan. - June Weak Interactions

Feb. - July Quantum Many-Body Computations for Cond. Matter Physics

S. Langer, Inst. for Theo. Phys., Univ. of California, Santa Barbara, CA 93106-4030, USA $8934111 / 8932431$

Feb. - Oct. European Centre for Theo. Studies in Nuclear Physics \& Related Areas

Feb. Fluctuations on Nuclear Dynamics

Feb. Mesons \& Baryons in Hadronic Matter

Feb. Nuclear Structure at Finite Temps.

March Monte Carlo Methods in Nuclear Structure Spring/Summer Investigating the Parton Structure of Nucleons \& Nuclei at Multi-GeV Energies July Hadron Structure \& QCD in Hard Processes

Oct. Parton Production \& Transport in the Quark-Gluon Plasma

Trento, Italy Somarive, 14, I-38050 Povo+ +39 (461) 881508 / 881699 preside @ itncisca.bitnet

\section{Centre for Chaos and Turbulence Studies}

at the

\section{University of Copenhagen and Denmark's Technical University}

has several theorist and/or experimentalist openings at a post-doctoral level, for research in classical and quantum chaos, nonlinear chemical dynamics, hydrodynamical and chemical turbulence, and complex systems in physics, chemistry and biology. The research is carried out at the Niels Bohr Institute, the Department of Chemistry at the University of Copenhagen, and the Physics Institute at the Technical University, often in close collaboration with other groups, such as the astrophysics, nuclear, particle and condensed matter physics, chemistry, biology and neural networks groups.

We are particularly interested in strengthening the experimental activity in connection with the well-established theoretical research.

Applications (CV together with description of research) should be sent before 12 December 1993 to: Dorte Glass, Secretary

CATS, Niels Bohr Institute

Blegdamsvej 17, DK-2100 Copenhagen $\varnothing$

Fax: +4531421016 - Phone: +4535325328 - E-mail: cats@ nbivax.dk

The applicant should also arrange for 3 letters of reference to be sent to the above address. Applications are coordinated with those to NORDITA and the Niels Bohr Institute, and need not be sent separately. 